\title{
1. Extended Data
}

\begin{tabular}{|c|c|c|c|}
\hline Figure \# & $\begin{array}{l}\text { Figure title } \\
\text { One sentence only }\end{array}$ & $\begin{array}{l}\text { Filename } \\
\text { This should be the name } \\
\text { the file is saved as when it } \\
\text { is uploaded to our system. } \\
\text { Please include the file } \\
\text { extension. i.e.: } \\
\text { Smith_ED_Fig1.jpg }\end{array}$ & $\begin{array}{l}\text { Figure Legend } \\
\text { If you are citing a reference for the first time in these legends, please include } \\
\text { all new references in the Online Methods References section, and carry on } \\
\text { the numbering from the main References section of the paper. }\end{array}$ \\
\hline Extended Data Fig. 1 & $\begin{array}{l}\text { Transcriptomic profiling } \\
\text { PBMCs stimulated with } \\
\text { live } C \text {. albicans or } C \text {. auris } \\
\text { and respective cell wall } \\
\text { components } \beta \text {-glucans and } \\
\text { mannans for } 4 \text { and } 24 \\
\text { hours. }\end{array}$ & $\begin{array}{l}\text { Ext_data_figure1.ep } \\
\mathrm{s}\end{array}$ & $\begin{array}{l}\text { a, Principal component analysis (PCA) performed on normalized } \\
\text { count data (normTransform, DESeq2) demonstrates the main } \\
\text { component introducing variance in the dataset is time (40\%), as } \\
\text { indicated by a clear split between the early 4-hour host induced } \\
\text { response (left, triangle) and the late } 24 \text {-hour response (right, circle). } \\
\text { To a lower extent (15\%), the second component introducing variance } \\
\text { appears to be inherent to the stimulus (color). b, At } 4 \text { hours, PCA } \\
\text { reveals a clear donor clustering (shape) irrespective of stimulus } \\
\text { (color), indicating the main variance in the early host response } \\
\text { reflects inter-individual differences (left). PCA on the late response, } \\
24 \text { hours, is predominantly influenced by the respective stimulus } \\
\text { (38\%, color), and to a lower extent by the donors (19\%, shape), } \\
\text { indicated by the scattering of stimuli together with a rough clustering } \\
\text { amongst donors. c, Pathway enrichment plot displaying the top } 20 \\
\text { enriched pathways for both C. albicans live and C. auris live (color) at } \\
24 \text { hours. Enrichment determined using Consensus PathDB, including } \\
\text { pathways as defined by KEGG and Reactome (shape), considering a p- } \\
\text { adjusted value < } 0.01 \text { (indicated as ' } q \text {-value') significant. Size of the } \\
\text { geometric points indicates the amount of DEG in relation to the } \\
\text { pathways' size. The exact q values and the data used to make this } \\
\text { figure can be found in Source Data Extended Data Fig.1. }\end{array}$ \\
\hline Extended Data Fig. 2 & Comparative LDH secretion, & Ext_data_figure2.ep & a, Assessment of Candida induced cell death of PBMCs after 24 hours \\
\hline
\end{tabular}




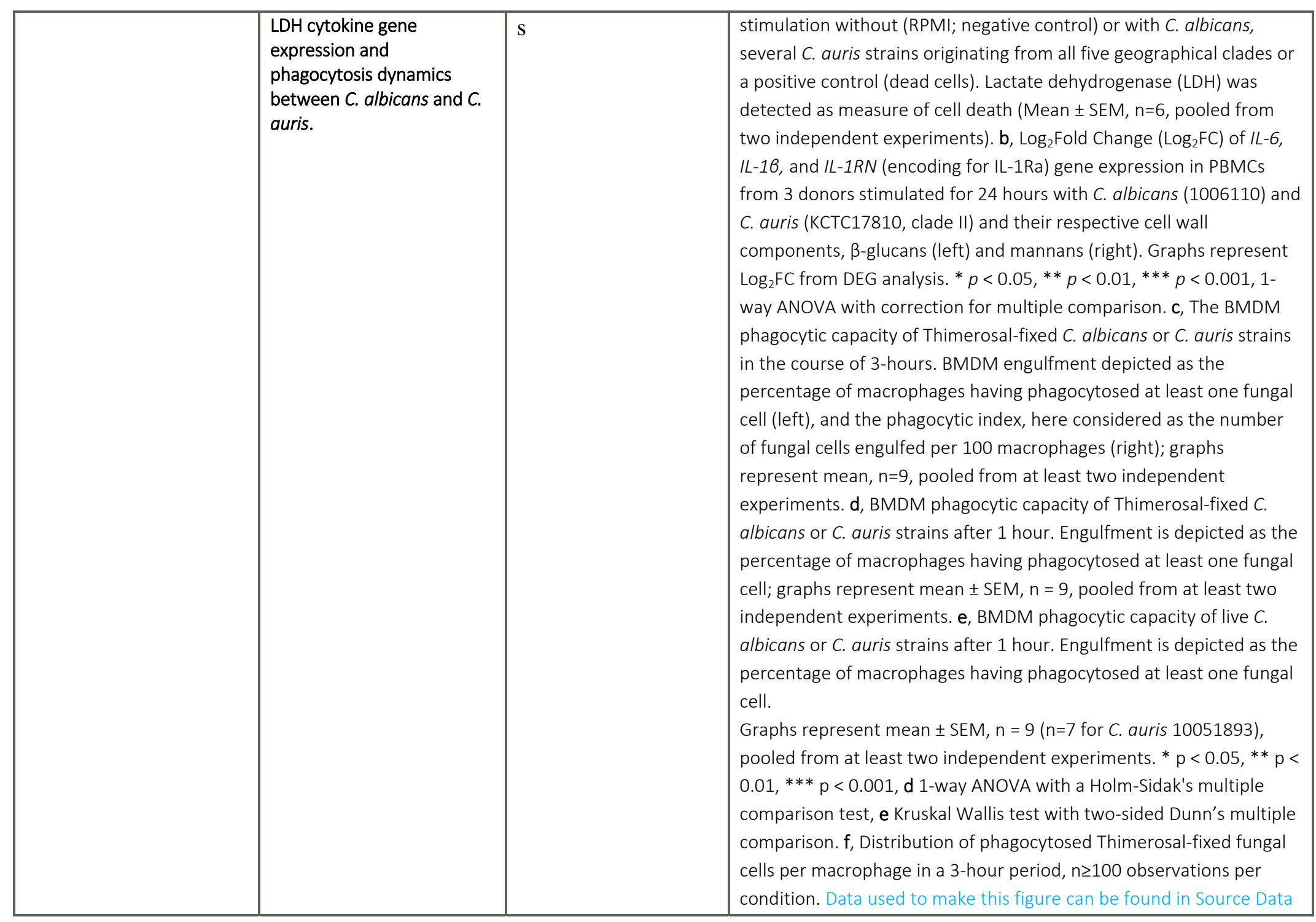




\begin{tabular}{|c|c|c|c|}
\hline & & & Extended Data Fig. 2. \\
\hline Extended Data Fig. 3 & $\begin{array}{l}\text { Relative } C \text {. auris induced } \\
\text { ROS production and heat- } \\
\text { sensitivity of the cell wall } \\
\text { components responsible for } \\
\text { the } C \text {. auris induced } \\
\text { cytokine production. }\end{array}$ & $\begin{array}{l}\text { Ext_data_figure3.ep } \\
\text { s }\end{array}$ & 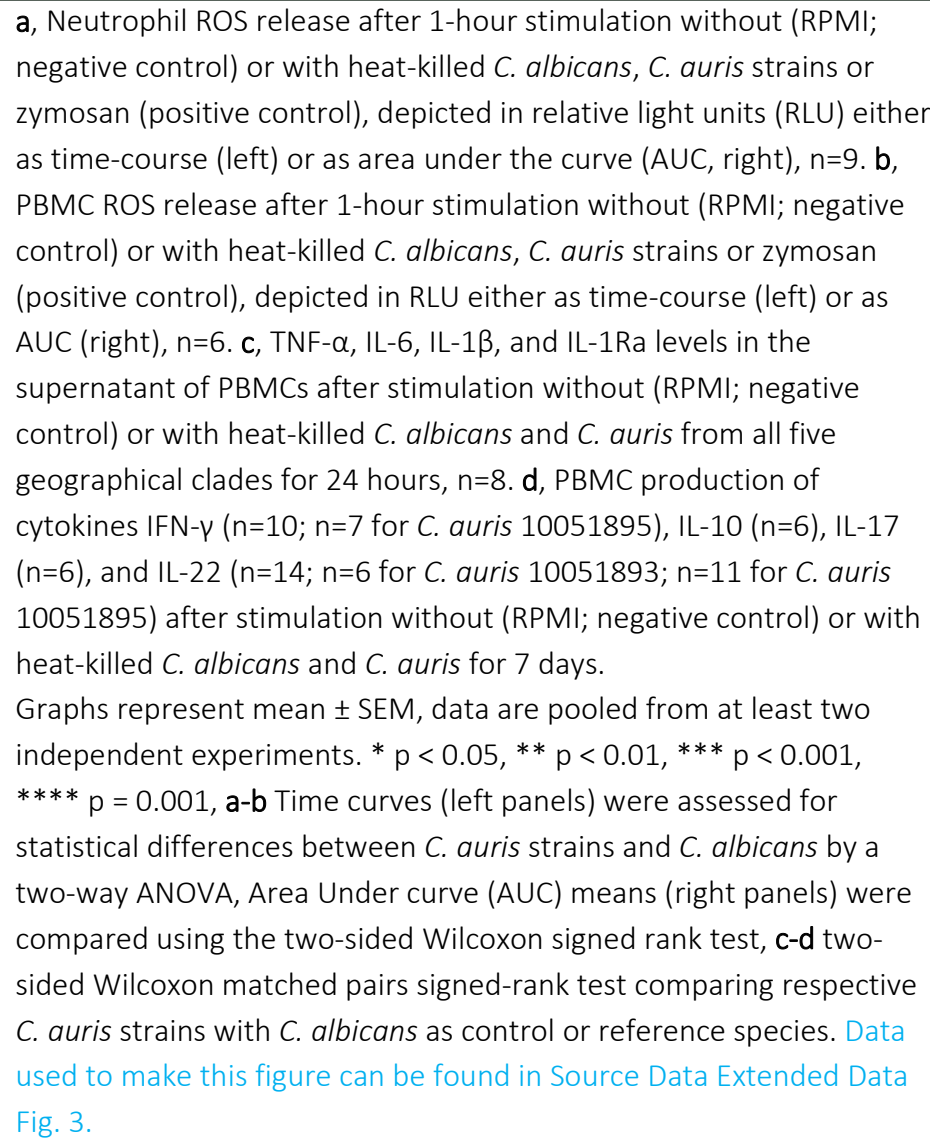 \\
\hline Extended Data Fig. 4 & $\begin{array}{l}\text { Transcriptional changes } \\
\text { induced by purified cell wall } \\
\text { components and their } \\
\text { respective exposure on } C \text {. } \\
\text { albicans and } C \text {. auris }\end{array}$ & $\begin{array}{l}\text { Ext_data_figure4.ep } \\
\text { s }\end{array}$ & $\begin{array}{l}\text { a, Heatmap displaying the } \text { Log }_{2} \text { Fold change (color scale) of the top } \\
50 \text { DEG of C. albicans live, for both Candida species and their cell wall } \\
\text { components, } \beta \text {-glucan and mannan, at } 4 \text { hour (left panel) and } 24 \\
\text { hours (right panel). b, Flow cytometry plot based on forward scatter } \\
\text { component (FSC) and side scatter component (SSC), demonstrating C. }\end{array}$ \\
\hline
\end{tabular}




\begin{tabular}{|c|c|c|c|}
\hline & surface. & & $\begin{array}{l}\text { auris strains are slightly smaller and of higher complexity than } C \text {. } \\
\text { albicans. c, Flow cytometry-based comparison of cell wall } \\
\text { components of } C \text {. albicans and } C \text {. auris strains. Mean fluorescent } \\
\text { intensity (MFI) of thimerosal-fixed Candida cells stained for Fc-Dectin- } \\
1 \text {, a marker for } \beta \text {-glucan (left), and ConA, a marker for mannans } \\
\text { (right). Graphs represent mean } \pm \text { SEM of the } 3 \text { means, each } \\
\text { performed with three replicates in three independent } \\
\text { measurements, }{ }^{*} p<0.05 \text {, Kruskall Wallis test with two-sided Dunn's } \\
\text { multiple comparison test was performed comparing the respective } C \text {. } \\
\text { auris strains with the two } C \text {. albicans reference strains. Data used to } \\
\text { make this figure can be found in Source Data Extended Data Fig. } 4 \text {. }\end{array}$ \\
\hline Extended Data Fig. 5 & $\begin{array}{l}\text { Evaluation of cytokine } \\
\text { production upon } C \text {. albicans } \\
\text { and } C \text {. auris mannan } \\
\text { stimulation. }\end{array}$ & $\begin{array}{l}\text { Ext_data_figure5.ep } \\
\text { s }\end{array}$ & 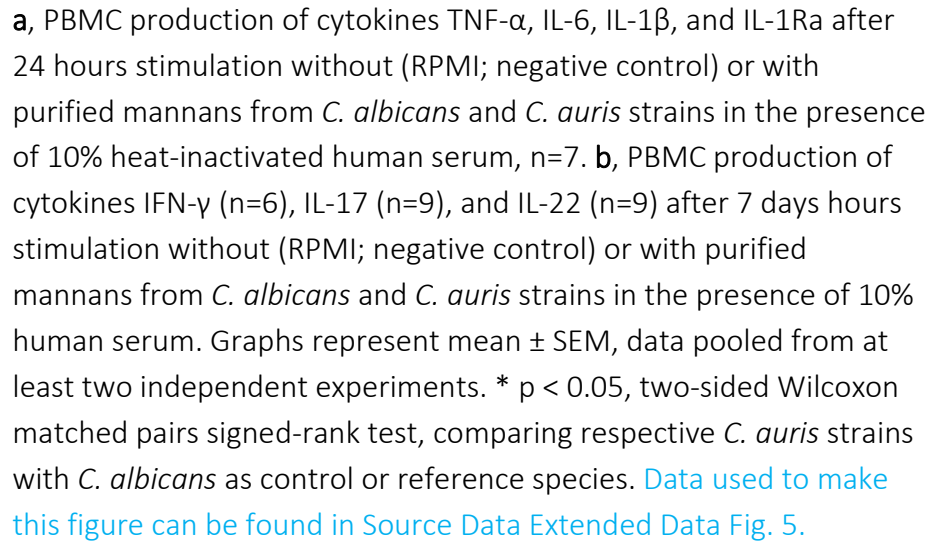 \\
\hline Extended Data Fig. 6 & $\begin{array}{l}\text { Cytokine levels in plasma } \\
\text { and organ homogenates } \\
\text { from C.albicans and } C \text {. } \\
\text { auris-infected mice. }\end{array}$ & $\begin{array}{l}\text { Ext_data_figure6.jp } \\
\text { g }\end{array}$ & 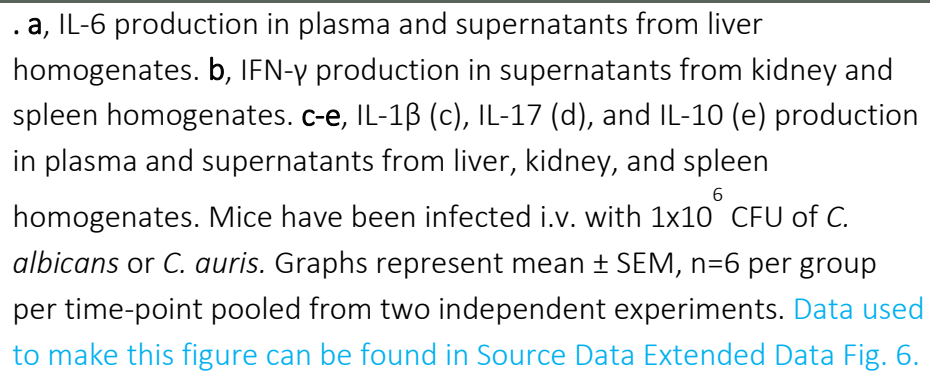 \\
\hline
\end{tabular}




\begin{tabular}{|l|l|l|l|}
\hline Extended Data Fig. 7 & $\begin{array}{l}\text { Applied gating strategies } \\
\text { across flow cytometry } \\
\text { experiments. }\end{array}$ & $\begin{array}{l}\text { Ext_data_figure7.ep } \\
\text { S }\end{array}$ & $\begin{array}{l}\text { a, Gating strategy for FITC-labelled Candida in PBMCs (linked to } \\
\text { Figure 2b). All events were plotted based on forward scatter (FS) and } \\
\text { side scatter (SS) characteristics. In the upper plot (2.1) the region of } \\
\text { cells positive for FITC-Candida was highlighted (green gate) while in } \\
\text { the bottom plot (2.2) CD14 positive cells are represented (red gate) } \\
\text { gated within the total PBMCs population (1). Within the CD14+ cells } \\
\text { selection, the amount of phagocytosed FITC positive Candida was } \\
\text { examined by plotting (3) the FITC signal against the CD14-PB450 } \\
\text { signal (blue gate) and the percentage of cells and mean fluorescent } \\
\text { intensity (MFI) were used for analysis. b, Gating strategy for } \\
\text { Thimerosal-fixed Candida cells stained for either } \beta \text {-glucan using Fc- } \\
\text { Dectin-1 or ConA as marker for mannans (linked to Figures S4c). }\end{array}$ \\
\hline 5
\end{tabular}

\section{Supplementary Information:}

\begin{tabular}{|l|l|l|l|}
\hline Item & Present? & $\begin{array}{l}\text { Filename } \\
\text { This should be the name the } \\
\text { file is saved as when it is } \\
\text { uploaded to our system, and } \\
\text { should include the file } \\
\text { extension. The extension must } \\
\text { be.pff }\end{array}$ & $\begin{array}{l}\text { A brief, numerical description of file contents. } \\
\text { i.e.: Supplementary Figures 1-4, Supplementary Discussion, and } \\
\text { Supplementary Tables 1-4. }\end{array}$ \\
\hline Supplementary Information & Yes & $\begin{array}{l}\text { SI_Supplementary_tab } \\
\text { les.pdf }\end{array}$ & Supplementary Tables 1-4 \\
\hline
\end{tabular}




\begin{tabular}{|l|l|l|l|}
\hline Type & $\begin{array}{l}\text { Number } \\
\text { If there are multiple files of the same type this } \\
\text { should be the numerical indicator. i.e. "1" for } \\
\text { Video 1, “2” for Video 2, etc. }\end{array}$ & $\begin{array}{l}\text { Filename } \\
\text { This should be the name the file is saved as } \\
\text { when it is uploaded to our system, and should } \\
\text { include the file extension. i.e.: Smith_ } \\
\text { Supplementary_Video_1.mov }\end{array}$ & $\begin{array}{l}\text { Legend or Descriptive Caption } \\
\text { Describe the contents of the file }\end{array}$ \\
\hline Supplementary Video & 1 & S1.mp4 & $\begin{array}{l}\text { C. auris is able to multiply within } \\
\text { phagosomes. }\end{array}$ \\
\hline Supplementary Video & & S2.mp4 & $\begin{array}{l}\text { C. auris accumulates in high } \\
\text { numbers within macrophages and } \\
\text { does not induce macrophage lysis. }\end{array}$ \\
\hline Supplementary Video & 2 & $\begin{array}{l}\text { C. auris cells are taken up } \\
\text { extensively into a subpopulation } \\
\text { of macrophages. }\end{array}$ \\
\hline Supplementary Video & 3 & S3.mp4 & $\begin{array}{l}\text { Phagocytosis of C. albicans } \\
\text { SC5314, macrophage lysis after 3 } \\
\text { hours. }\end{array}$ \\
\hline
\end{tabular}

\section{Source Data}

Complete_Bruno_repo rting_summary.pdf

\section{B. Additional Supplementary Files}




\begin{tabular}{|l|l|l|}
\hline Figure & $\begin{array}{l}\text { Filename } \\
\text { This should be the name the file is saved as when } \\
\text { it is uploaded to our system, and should include } \\
\text { the file extension. i.e. } \\
\text { Smith_SourceData_Fig1.xls, or Smith_ } \\
\text { Unmodified_Gels_Fig1.pdf }\end{array}$ & $\begin{array}{l}\text { Data description } \\
\text { i.e.: Unprocessed Western Blots and/or gels, Statistical Source Data, etc. }\end{array}$ \\
\hline Source Data Fig. 1 & SD_Main_Fig1.xlsx & Supporting data for Fig. 1 \\
\hline Source Data Fig. 2 & SD_Main_Fig2.xlsx & Supporting data for Fig. 2 \\
\hline Source Data Fig. 3 & SD_Main_Fig3.xlsx & Supporting data for Fig. 3 \\
\hline Source Data Fig. 5 & SD_Main_Fig5.xlsx & Supporting data for Fig. 5 \\
\hline Source Data Fig. 6 & SD_Main_Fig6.xlsx & Supporting data for Fig. 6 \\
\hline $\begin{array}{l}\text { Source Data Extended } \\
\text { Data Fig. } 1\end{array}$ & SD_Ext_Data_Fig1.xlsx & Supporting data for Extended Data Fig. 1. \\
\hline $\begin{array}{l}\text { Source Data Extended } \\
\text { Data Fig. } 2\end{array}$ & SD_Ext_Data_Fig2.xlsx & Supporting data for Extended Data Fig. 2. \\
\hline $\begin{array}{l}\text { Source Data Extended } \\
\text { Data Fig. } 3\end{array}$ & SD_Ext_Data_Fig3.xlsx & Supporting data for Extended Data Fig. 3. \\
\hline $\begin{array}{l}\text { Source Data Extended } \\
\text { Data Fig. } 4\end{array}$ & SD_Ext_Data_Fig4.xlsx & Supporting data for Extended Data Fig. 4. \\
\hline $\begin{array}{l}\text { Source Data Extended } \\
\text { Data Fig. } 5\end{array}$ & SD_Ext_Data_Fig5.xlsx & Supporting data for Extended Data Fig. 5. \\
\hline $\begin{array}{l}\text { Source Data Extended } \\
\text { Data Fig. } 6\end{array}$ & SD_Ext_Data_Fig6.xlsx & Supporting data for Extended Data Fig. 6. \\
\hline
\end{tabular}




\section{Host immune response against the emerging fungal pathogen Candida auris:} transcriptional and functional insights Mariolina Bruno ${ }^{\# 1}$, Simone Kersten ${ }^{\# 1,2}$, Judith M. Bain ${ }^{3}$, Martin Jaeger ${ }^{1}$, Diletta Rosati ${ }^{1}$, Michael D. Kruppa ${ }^{4}$, Douglas W. Lowman ${ }^{4}$, Peter J. Rice, ${ }^{5}$,Bridget Graves ${ }^{4}$, Ma Zuchao ${ }^{4}$, Y. Ning Jiao $^{4}$, Anuradha Chowdhary ${ }^{6}$, George Renieris ${ }^{7}$, Frank L. van de Veerdonk ${ }^{1,8}$, Bart-Jan Kullberg ${ }^{1,8}$, Evangelos J. Giamarellos-Bourboulis', Alexander Hoischen ${ }^{1,2}$, Neil A. R. Gow ${ }^{3,9}$,

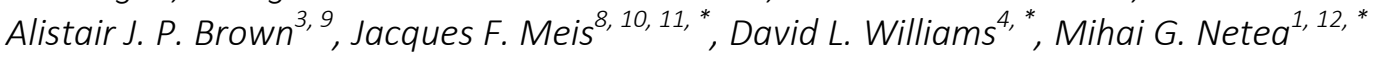

${ }^{1}$ Department of Internal Medicine, Radboud Institute for Molecular Life Sciences, Radboud University Medical Center, Nijmegen, the Netherlands

2 Department of Human Genetics, Radboud Institute for Molecular Life Sciences, Radboud University Medical Center, Nijmegen, the Netherlands

${ }^{3}$ Medical Research Council Centre for Medical Mycology, University of Aberdeen, Foresterhill, Aberdeen, UK

${ }^{4}$ Departments of Surgery, Biomedical Sciences and Center of Excellence in Inflammation, Infectious Disease and Immunity, Quillen College of Medicine, East Tennessee State University, Johnson City, TN, USA

${ }^{5}$ Department of Clinical Pharmacy, Skaggs School of Pharmacy and Pharmaceutical Sciences, University of Colorado, Aurora, CO, USA

${ }^{6}$ Department of Medical Mycology, Vallabhbhai Patel Chest Institute, University of Delhi, New Delhi, India

${ }^{7}$ 4th Department of Internal Medicine, National and Kapodistrian University of Athens, Medical School, Athens, Greece.

${ }^{8}$ Center of Expertise in Mycology, Radboud University Medical Center and Canisius Wilhelmina Hospital, Nijmegen, the Netherlands

${ }^{9}$ MRC Centre for Medical Mycology, University of Exeter, Geoffrey Pope Building, Stocker Road, Exeter EX4 4QD, UK.

${ }^{10}$ Bioprocess Engineering and Biotechnology Graduate Program, Federal University of Paraná, Curitiba, PR, Brazil

${ }^{11}$ Department of Medical Microbiology and Infectious Diseases, Canisius Wilhelmina Hospital, Nijmegen, the Netherlands

${ }^{12}$ Department for Genomics \& Immunoregulation, Life and Medical Sciences Institute (LIMES), University of Bonn, Germany

${ }^{\#}$ These authors contributed equally

*These authors share senior authorship

Corresponding author:

60 Mihai G. Netea

61 Department of Internal Medicine (463) and Radboud Center for Infectious Diseases (RCI), 62 Radboud University Nijmegen Medical Centre, Geert Grooteplein 8, Nijmegen 6500 HB, the 63 Netherlands. mihai.netea@radboudumc.nl 
65 ABSTRACT (MAX 150 words)

66 Candida auris is amongst the most important emerging fungal pathogens, yet mechanistic

67 insights in its immune recognition and control are lacking. Here, we integrate transcriptional

68 and functional immune cell profiling to uncover innate anti-C. auris defense mechanisms. $C$.

69 auris induces a specific transcriptome in human mononuclear cells, a stronger cytokine

70 response compared to C. albicans, but a lower macrophage lysis capacity. C. auris-induced

71 innate immune activation is mediated through recognition of C-type lectin receptors, mainly

72 elicited by structurally unique C. auris mannoproteins. In in-vivo experimental models of

73 disseminated candidiasis, C. auris was less virulent than C. albicans. Collectively, these results

74 demonstrate that $C$. auris is a strong inducer of innate host defense and identify possible

75 targets for adjuvant immunotherapy. 


\section{INTRODUCTION}

78 Candida auris is an important emerging fungal pathogen that was first described in 2009, and has,

79 since then, spread across six continents as a causative microorganism of hospital-acquired infections ${ }^{1}$.

80 For several reasons, $C$. auris is among the most challenging of emerging human pathogens identified

81 in the last decade. It is highly resistant to many of the commonly used antifungal drugs ${ }^{1}$ and, within a

82 few years, it has rapidly spread worldwide ${ }^{2,3}$ through the nearly simultaneous (but independent)

83 emergence of four distinct phylogeographical clades ${ }^{4}$. Recently, a potential fifth clade has been

84 described in Iran ${ }^{5}$. Every major clade except for clades II and V has been linked to outbreaks with

85 invasive infection ${ }^{6}$. On the contrary, clade II generally shows antifungal susceptibility and has a

86 propensity for ear infections. Similar to clade II, clade V isolate was recovered from ear. Clade III is

87 associated with bloodstream infections and, together with clade II, tends to form large cell aggregates

$88{ }^{7}$. This has been linked with a reduced virulence in a Galleria mellonella infection model ${ }^{8}$.

89 C. auris poses difficulties in routine microbiological identification ${ }^{9,10}$ and it is challenging to

90 eradicate in healthcare settings ${ }^{11-14}$. This is due to its strong capacity to colonize skin, its

91 transmittance via patient-to-patient route or contaminated fomites, and its high survival capacity on

92 plastic surfaces and in the hospital environment ${ }^{15}$. The risk factors for C. auris infections are generally

93 similar to those for other types of Candida infections, such as prolonged hospitalization, use of central

94 venous catheters, abdominal surgery and exposure to broad-spectrum antibiotics or antifungals ${ }^{16}$.

95 However, due to its acquired resistance to many antifungal drugs, the overall crude mortality rate of

96 C. auris candidemia is high, ranging from $30 \%$ to $60 \%$, with infections typically occurring several weeks

97 (10-50 days) after admission ${ }^{4,12,17,18}$. Echinocandins are currently recommended by the CDC as empiric

98 treatment of $C$. auris infections, although resistance has been reported. Several new therapeutic

99 alternatives, such as fosmanogepix ${ }^{19,20}$, ibrexafungerp ${ }^{21-23}$ and rezafungin ${ }^{24,25}$ are currently under

100 clinical investigation.

101 Considering the importance of $C$. auris as an emerging human pathogen, it is imperative to

102 understand the host defense mechanisms. This is particularly true given the high resistance of this 
103 fungus to anti-mycotic drugs, which makes it a prime candidate for the development of host-directed

104 therapy (i.e. immunotherapy). However, almost nothing is known regarding the host immune

105 response against $C$. auris. Host defense against Candida species is dependent on a finely tuned

106 interplay of innate and adaptive immune responses. A first physical barrier consists of the skin and

107 mucosa. The second barrier, represented by the innate immune system, is largely dependent on the

108 recognition of evolutionarily conserved fungal cell wall components (pathogen-associated molecular

109 patterns, PAMPs) by innate immune cells such as monocytes, macrophages and neutrophils. In turn,

110 the release of proinflammatory cytokines, combined with antigen-presentation activity of myeloid

111 cells, is crucial for shaping the adaptive immunity, representing a third, long-term barrier against

112 fungal infection ${ }^{26}$.

113 The Candida cell wall is divided into an outer layer of highly mannosylated proteins

114 (mannoproteins) and an inner layer, mainly comprised of $\beta(1 \rightarrow 3)$ and $\beta(1 \rightarrow 6)$-glucans and chitin ${ }^{27}$.

115 These PAMPs are recognized by various pattern recognition receptor (PRRs) on the surface of immune

116 cells: C-type lectin receptors (CLRs) such as Dectin-1, Dectin-2, macrophage mannose receptor (MMR),

117 Mincle (macrophage-inducible C-type lectin), DC-SIGN (dendritic cell specific intercellular adhesion

118 molecule-3-grabbing non-integrin) and Toll-like Receptors (TLRs), especially TLR2 and TLR4 ${ }^{28}$.

119 Coordinated engagement of PRRs results in the activation of innate immune effector mechanisms

120 such as phagocytosis, reactive oxygen species (ROS) release and production of pro- and anti-

121 inflammatory cytokines. In turn, together with the antigen-presentation activity of myeloid cells, the

122 release of pro-inflammatory cytokines shapes the adaptive immune response ${ }^{26}$.

123 While the antifungal host defense mechanisms have been extensively studied for C. albicans,

124 little is known about the host immune response against $C$. auris. Almost all multi-drug resistant $C$.

125 auris strains are susceptible to killing by the salivary antimicrobial peptide Histatin $5(\mathrm{Hst}-5)^{29}$, while

126 Johnson and colleagues showed that neutrophil recruitment and formation of neutrophils

127 extracellular traps (NETs) were lower for $C$. auris than C. albicans ${ }^{30}$. Recently it was reported that $C$.

128 albicans, C. tropicalis, C. guilliermondii, C. krusei and C. auris differentially stimulate cytokine 
production in peripheral blood mononuclear cells (PBMCs) ${ }^{31}$, but little is known regarding the

130 particularities of these responses and the mechanisms mediating them. Considering the knowledge

131 gap in our understanding of anti-C. auris host defense mechanisms, we set out to comprehensively

132 assess the mechanisms through which innate immune cells recognize $C$. auris, initiate innate

133 antifungal immune responses, and protect the host against $C$. auris infection. This mechanistic insight

134 into C. auris host interactions is instrumental for the development of novel host-directed approaches

135 for the treatment of severe C. auris infections and, thereby, improve patient outcomes.

\section{RESULTS}

138 Common and specific transcriptome signatures induced by $C$. albicans and C. auris in human immune

139 cells

140 To gain a broad overview of the host immune response against $C$. auris, RNA sequencing was

141 performed on PBMCs from three healthy donors that were exposed to either live $C$. albicans or $C$.

142 auris for 4 or 24 hours. Due to the high genome-wide nucleotide identity across C. auris clades I-IV

$143(98.7 \%)^{32}$, the analysis of the C. auris reference strain KCTC171810 (clade II) is expected to provide

144 valuable insight into generic $C$. auris induced host responses. This C. auris reference strain was

145 compared to C. albicans 10061110, which, to date, remains the most common cause of mucosal and

146 systemic candidiasis ${ }^{33}$.

147 Principle component analysis (PCA) of the normalized PBMC RNAseq dataset revealed that the

148 majority of the variance in the experiment as a whole was time-dependent, as demonstrated by a

149 clear separation of the 4-hour and 24-hour stimulation time points (Extended Data Fig. 1a).

150 Comparison of the stimulated and non-stimulated samples at the 4-hour time point indicates that a

151 limited response was induced early on. Moreover, stimulus clustering at 4 hours suggests this short-

152 term response was similar in C. auris and C. albicans (Extended Data Fig. 1a). At the 4-hour time point,

153 clustering of the donors irrespective of stimulus indicates that inter-individual differences underpin 
154 the observed variance (Extended Data Fig. 1b, left). As PBMC donors were considered biological

155 replicates, comparison of the average PBMC response to their control condition revealed significant

156 overlap in the 4-hour host response between C. albicans and C. auris. With 71 differentially expressed

157 genes (DEG; fold change $\geq 2$ and $p$-adjusted value $<0.01$ ) upregulated by both Candida species, the

158 respective overlap ranges from $67 \%$ of the total number of DEG for C. albicans (71 / 109) to $95 \%$ of

159 the total number of DEG for $C$. auris (71 / 79).

160 In contrast, at 24 hours, the response is primarily stimulus-driven (Extended Data Fig. 1b,

161 right), as indicated by the scattering of donor responses dependent on pathogen exposure. After $24 \mathrm{~h}$

162 of stimulation, the common C. auris and C. albicans-induced host response increased to 243 DEG

163 (Figure 1a), in turn accounting for 55\% of the total number of DEG for C. albicans (243 / 442) and 50\%

164 of the total number of DEG for C. auris (243 / 484). This late shared response between both Candida

165 species was consistent with the observation that the 24-hour induced PBMC transcriptomes were

166 more stimulus-specific (Extended Data Fig. 1b). Pathway enrichment analysis revealed that the 4-hour

167 Candida intrinsic response was delineated by a common activation of the CC and CXC chemokines

168 (Supplementary Table 1). In contrast, the 24-hour PBMC transcriptomic response was characterized by

169 a broader upregulation of chemokines, interleukins (IL), tumor necrosis factor and their receptors

170 (Figure 1a and Supplementary Table 2).

171 The substantial activation of glucose, fructose and mannose metabolism was unique to the $C$.

172 albicans-induced transcriptional response of PBMCs at 24 hours. Conversely, the DEG that were more

173 strongly induced upon PBMC exposure to C. auris appeared to be linked to type I and II interferons, as

174 well as antiviral mechanisms triggered via IFN-stimulated genes, including the ISG15 immune

175 mechanisms (Figure 1a and Supplementary Table 2). Collectively, these data show that C. albicans and

176 C. auris are potent activators of the host immune system, and they are not only able to activate

177 common transcriptional responses, but also induce pathways specific to each pathogen.

178 
180 One-third of the top 15 enriched pathways, based on those DEG that were unique for $C$. auris, were

181 also found to be enriched in the common Candida response (Figure 1a). This was not the case for $C$.

182 albicans, indicating that $C$. auris has the ability to upregulate more genes in these pathways compared

183 to C. albicans. Most pronounced within these unique DEG were distinct interleukins such as IL1RN

184 (encoding for IL-1Ra), IL10, IL19, IL26 and IL27, as well as interferon (IFN) associated genes, e.g. STAT2,

185 DDX58, EIF2AK2, OAS2, OAS3, IFIT2, IFIT3, IFIT35 and IFITM1 (Supplementary Table 2). Furthermore,

186 DEG were more potently induced in response to C. auris than C. albicans (Student's T-test, p-value of

187 0.003). An additional pathway enrichment analysis on all the upregulated DEG confirmed that the

188 total number of DEG for mutually enriched pathways was higher when the PBMCs were stimulated

189 with C. auris rather than with C. albicans (Extended Data Fig. 1c). Collectively, the broader and

190 stronger induction of DEG by C. auris resulted in higher enrichment scores (q-value) for corresponding

191 pathways in comparison to C. albicans, suggesting that $C$. auris is a more potent trigger of the host

192 response.

193 With the transcriptomic analysis suggesting cytokine signaling to be at the core of the host

194 response, we aimed to verify these observations at the protein level. For this, the cytokine production

195 ability of PBMCs isolated from healthy volunteers was assessed following 24-hour exposure to three

196 different live clinical isolates for each Candida species, all cultured under similar conditions. As a

197 measure of Candida induced cytotoxicity, the detection of lactate dehydrogenase (LDH) release

198 revealed that PBMC viability after 24 hours was not impacted (Extended Data Fig. 2a). With the

199 exception of the anti-inflammatory cytokine IL-1Ra, PBMCs exposed for 24 hours to both clinical

200 isolates of $C$. auris produced significantly higher amounts of the pro-inflammatory cytokines TNFa, IL-

201 6, and IL-1 $\beta$ compared to the C. albicans-stimulated PBMCs (Figure 1b). Moreover, to test whether this

202 observation is clade-dependent or reflects the general C. auris response, cytokine production was

203 assessed upon 24-hour stimulation with different $C$. auris strains originating from the five different

204 clades. While showing variation between C. auris strains in cytokine production (Figure 1c), a similar 
pattern between pro-inflammatory cytokines was observed. Of note, $C$. auris clade V induced similar

206 levels of pro-inflammatory cytokines and significantly lower IL-1Ra levels compared to C. albicans. C.

207 auris clades II and III induced cytokine production moderately, but this was still significantly higher

208 compared to C. albicans. C. auris clades I and IV present as extremely potent inducers of pro-

209 inflammatory cytokines. Regardless of this clade dependent variability, all C. auris clades except for

210 Clade $V$ were observed to drive a significantly enhanced pro-inflammatory cytokine response

211 compared to C. albicans (Figure 1c). However, at the transcriptional level, PBMCs stimulated with C.

212 auris showed only a trend towards a stronger induction of IL-6 ( $\log _{2}$ FC $8.41 \pm$ IfCSE 1.4$)$ and IL-16

213 ( $\log _{2} \mathrm{FC} 6.45 \pm$ IfCSE 1.29) expression levels than upon exposure to $C$. albicans ( $\log _{2} \mathrm{FC}=7.58 \pm$ IfCSE

2141.37 for IL-6; $\log _{2}$ FC $5.59 \pm$ IfCSE 1.31 for IL-16) (ns, Extended Data Fig. 2b).

215

216 C. auris replicates faster than C. albicans in vivo, leading to altered multiplicity of infection (MOI), but

217 does not cause macrophage lysis

218 Killing of Candida by professional phagocytes of the innate immune system, such as monocytes,

219 macrophages or dendritic cells, is an important line of defense at the site of infection. In order to

220 study the differences in phagocytosis dynamics of professional phagocytes between $C$. auris and $C$.

221 albicans, we employed live-cell video microscopy coupled with dynamic image analysis of bone

222 marrow-derived macrophages (BMDMs). Phagocytosis capacity was assessed by combining BMDMs

223 with live and thimerosal-killed C. auris and C. albicans strains at an intended MOI of 3:1, yeast cells per

224 macrophage. By including fixed yeasts, we were able to assay phagocytosis in the absence of rapid

225 adaptive changes in the composition of the Candida cell wall. Results are expressed as percentage of

226 phagocytic BMDM (\% uptake), indicating the percentage of macrophages having phagocytosed at

227 least one fungal cell. In addition, we assayed the Phagocytic Index which is defined as the number of

228 fungal cells engulfed and fully inside the phagosome per 100 macrophages (by excluding the fungal

229 cells adhering but not internalized). No significant differences in C. auris and C. albicans phagocytosis

230 (\% uptake) were observed for fixed Candida (Extended Data Fig. 2c). There was a trend towards a 
231 higher phagocytic index in BMDMs at later time points (after the second hour) for both of the fixed $C$.

232 auris strains compared to C. albicans strains (Extended Data Fig. 2c), possibly because fungal cells tend

233 to be phagocytosed in clusters. However, C. auris strain 10051893 had a lower phagocytosis efficiency

234 after 1 hour compared to C. albicans SC5314 (Extended Data Fig. 2d). Focusing on live strains, we

235 observed that the phagocytic index of both $C$. auris strains gradually increases in time, to a greater

236 extent than the C. albicans strains (Figure 2a). In order to evaluate the phagocytosis dynamics using

237 human cells, we incubated human PBMCs stimulated for 15, 30 and 120 minutes (2 hours) with

238 thimerosal-fixed FITC-labeled C. albicans 10061110 and C. auris KCTC17810, and then calculated the

239 percentage of FITC-positive cells as well as the mean fluorescence intensity (MFI) in the CD14

240 population. C. auris showed a significantly higher rate of phagocytosis and MFI at all the time points

241 measured compared to C. albicans (Figure 2b).

242 Using real-time live cell microscopy, live C. auris cells were observed to bud repeatedly outside

243 the macrophages, with a doubling time of $\sim 1$ hour. The $C$. auris budding rate decreased following

244 phagocytic engulfment, although cells continued to multiply within phagosomes (Supplementary Video

245 1). Of interest, C. auris 10051895 accumulated in high numbers within macrophages, indicating that

246 the starting MOI had exceeded the intended initial 3:1 ratio (Supplementary Video 2). This triggered

247 our interest to estimate the actual $\mathrm{MOI}$ ratio at the start of image acquisition. Due to the time elapsed

248 between the counting of Candida in each sample and the image acquisition, the starting 3:1 ratio for

249 C. auris rose up to 7:1, presumably due to ongoing budding, despite the fact that samples were stored

250 at $4^{\circ} \mathrm{C}$ in PBS until the live imaging commenced. In contrast, the MOls for live $C$. albicans as well as for

251 the fixed strains remained around the desired target of 3:1 yeast:macrophage.

252 The elevated MOls for $C$. auris may be a contributing factor to the trend for higher phagocytic

253 index achieved at 3 hours for live strain 10051895 (Figure 2a). However, an elevated starting MOI for

254 C. auris 10051893 did not enhance phagocytosis, as the live form of this strain was poorly recognized

255 by BMDMs, showing a significantly lower percentage of uptake as compared to C. albicans 10061110 
256 (Extended Data Fig. 2e). From the representative movies recorded, we quantified the distribution of

257 yeast per individual macrophage after 3 hours and found that for both $C$. auris strains, there was a

258 tendency for some macrophages to engulf many fungal cells, yet for other macrophages to engulf

259 none. This phenomenon was less surprising for the Candida experiments using live microorganisms

260 (Figure 2c), because C. auris continues to divide prior to and during the phagocytosis experiment.

261 However, the fixed strains also displayed this varied distribution, with both strain of $C$. auris being

262 phagocytosed in large numbers by some macrophages (Extended Data Fig. 2f). Supplementary Video 3

263 shows that $C$. auris cells are taken up extensively into a subpopulation of macrophages, but despite

264 the vast burden, these phagocytes continue to move around in pursuit of further fungal targets

265 (Supplementary Video 3).

266 Finally, macrophage lysis was determined following engulfment of live Candida and it emerged

267 that the $C$. auris strains examined were significantly less able to kill macrophages after 3 hours than $C$.

268 albicans 10061110, but not compared to C. albicans SC5314 (Figure 2d, Supplementary Video 4),

269 despite having a comparable (or greater, in the case of $C$. auris 10051895) phagocytic index. These

270 findings demonstrate that $C$. auris is differentially recognized by phagocytic BMDMs and internalized

271 with a higher phagocytic index compared to C. albicans but is not able to induce lysis of the phagocytic

272 cells.

273

274 Host immune response upon $C$. auris exposure is mediated by heat-sensitive cell wall

275 components.

276 Variability in the cell surface structures, such as differentially expressed mannoproteins or altered $\beta$ -

277 glucan exposure between the $C$. auris and C. albicans cell walls, could account for the differential

278 cytokine responses triggered by these pathogens. To elucidate whether this might account for the

279 observed differences in cytokine induction, the C. auris and C. albicans strains were subjected to heat-

280 killing. This approach facilitates disruption of the outer layer of the Candida cell wall ${ }^{34}$, in turn

281 enhancing $\beta$-glucan exposure ${ }^{35,36}$. The heat-killed strains were used to stimulate PBMCs for 24 hours 
and 7 days. Since the production of reactive oxygen species (ROS) can positively contribute to immune

283 responses ${ }^{37}$, in addition to PBMCs cytokine production, ROS release in both neutrophils and PBMCs

284 was assessed during 1 hour stimulation with serum-opsonized heat-killed C. auris and C. albicans

285 strains. The area under the curve (AUC) was calculated to examine quantitative differences in ROS

286 release.

287 In neutrophils both the ROS release over time and the total amount of production (AUC) were

288 significantly lower following C. auris stimulation, compared to C. albicans (Extended Data Fig. 3a). In

289 PBMCs, although the time-course luminescence was significantly decreased in C. auris-stimulated

290 cells, only C. auris 10051893 induced a significantly lower total ROS production than C. albicans

291 (Extended Data Fig. 3b).

292 Unexpectedly, compared to C. albicans, the cytokine response was almost completely

293 abrogated after PBMC stimulation with heat-killed C. auris for 24 hours (Figure 2e). This phenomenon

294 was observed for all C. auris clades (Extended Data Fig. 3c), subsequently marking this characteristic

295 for the general C. auris induced host immune response. In addition, after 7 days, the production of

296 IFN- $\gamma$ and IL-17 by PBMCs stimulated with heat-killed C. auris was significantly lower compared to

297 heat-killed C. albicans (Extended Data Fig. 3d). Hence, we reasoned that a heat sensitive component of

298 the cell wall might be responsible for most of the increased cytokine induction by C. auris.

299

300 Mannans drive the host response to $C$. auris

301 We attempted to unravel the contribution of the different fungal cell wall components to the

302 activation of host responses by $C$. auris, compared to C. albicans. PBMCs were exposed to the purified

303 cell wall components, $\beta$-glucans and mannans, from both Candida species, and the transcriptional

304 responses of the stimulated immune cells were assessed by RNA sequencing. The species-specific cell

305 wall contribution was assessed by comparison of the number of shared DEG upon exposure to the

306 different cell wall components and expressed as proportion of the respective live stimulus. 
308 to explain around $82 \%$ and $57 \%$ of the respective live responses of $C$. albicans (89 / 109) and C. auris

309 (45 / 79) (Figure 3a). The $\beta$-glucans from each species resulted in similar PBMC gene expression

310 profiles (Extended Data Fig. 4a). Although the relative contribution of $C$. albicans $\beta$-glucan decreases to

311 approximately 13\% (55 / 442) in the late phase, 24 hours after stimulation, they remain able to

312 upregulate several C. albicans' top 50 DEG, leaving them a main contributor of the evoked response in

313 the live setting (Figure 3a and Extended Data Fig. 4a). In contrast, $\beta$-glucans from C. auris failed to elicit

314 a response analogous to the live $C$. auris exposure, explaining only a mere $2 \%(10 / 484)$ of the live $C$.

315 auris-induced response. Conversely, however, mannans from C. auris stimulated 28\% (136 / 484) of

316 the evoked transcriptional response to live $C$. auris cells. Moreover, C. auris mannan seemed to

317 outperform C. albicans $\beta$-glucan in relation to the top 50 DEG of $C$. albicans, displaying an induction

318 pattern similar to its live setting (Extended Data Fig. 4a). Overall, these results indicate that the host

319 recognition and subsequent initiation of downstream responses against C. albicans is mainly

320 dependent on $\beta$-glucan. For $C$. auris, early 4-hour stimulation of PBMCs is mainly mediated by $\beta$ -

321 glucan, whereas mannans are fundamental for orchestrating the $C$. auris-specific host response at

322 later time points (24 hours).

323 Our data suggest a stronger $C$. auris host immune response and a differential role in gene

324 expression between $C$. albicans and $C$. auris cell wall components. Therefore, to investigate the

325 differences in cell wall structure between C. auris and C. albicans, we first compared forward (FSC)

326 and side (SSC) light scatter of fungal cells using flow cytometry. In line with what has previously been

327 described ${ }^{23}$, we found that the $C$. auris strains have a smaller average cell size compared to $C$.

328 albicans. Of the C. auris strains, strain 10051893 shows more complexity/granularity (higher SSC) than

329 strain 10051895 (Extended Data Fig. 4b).

$330 \quad$ Next, we measured $\beta$-glucan exposure on the fungal cell surface by flow cytometry on

331 thimerosal-fixed Candida cells stained with Fc-Dectin-1. C. auris 10051893 displayed significantly

332 reduced exposure of $\beta$-glucan as compared to C. albicans SC5314 (Extended Data Fig. 4c). At the gene 
expression level, $C$. auris $\beta$-glucan ( $\log _{2} \mathrm{FC} 2.9 \pm$ IfCSE 0.6 ) tended to induce less effectively IL-1Ra as

334 compared to $\beta$-glucan isolated from C. albicans ( $\log _{2}$ FC $4.5 \pm$ IfCSE 0.6) (ns, Extended Data Fig. 2b). At

335 the cytokine level, though large variation between different strains was observed, no significant

336 differences in cytokine production of PBMCs stimulated with purified $\beta$-glucans from $C$. auris

337 compared to $C$. albicans were found for TNF $\alpha$ and IL-1 $\beta$, while there was significantly lower IL-6

338 production in response to $\beta$-glucan from C. auris $10031160, C$. auris $10051256, C$. auris $10051263, C$.

339 auris 10051522 and C. auris 10051252 when compared to C. albicans $\beta$-glucan. Interestingly, $\beta$-glucan

340 from C. auris strains, except for C. auris 10051522, C. auris 10051244 and C. auris 10051252, induced

341 a significantly lower IL-1Ra production compared to C. albicans $\beta$-glucan (Figure 3b). Moreover,

342 similarly to C. albicans $\beta$-glucan, C. auris $\beta$-glucan synergistically boosted Pam3Cys (TLR2 agonist)-

343 induced IL-1 $\beta$ production in PBMCs, as well as TNF $\alpha$ and IL-6 production (Figure 3c).

$344 \quad$ Having ruled out a major role for $\beta$-glucans in explaining the difference in cytokine stimulation 345 induced by C. auris and C. albicans, we assessed the role of glycosylated mannoproteins from the 346 fungal cell wall ${ }^{34}$. Examination of mannan exposure, by staining thimerosal-fixed Candida cells with

347 Concanavalin A (ConA), revealed a relatively low level of exposure of surface mannans in C. auris

348 strains. This difference was significant for C. auris 10051893 as compared with C. albicans 10061110

349 (Extended Data Fig. 4c). However, C. auris mannans were observed to significantly induce the gene

350 expression of pro-inflammatory cytokines $I L-6($ padj $=0.0001)$ and $I L-16($ padj $=0.0003)$ compared to

351 those of C. albicans (Extended Data Fig. 2b).

352 In line with these observations, mannans from all eight $C$. auris strains induced a significantly

353 higher cytokine production than C. albicans mannans 24 hours after stimulation of PBMCs, both pro-

354 inflammatory and anti-inflammatory (Figure 3d). Except for IL-1Ra production, opsonization by human

355 serum was necessary for mannan-induced production of cytokines (Extended Data Fig. 5a). After 7

356 days of stimulation, no significant differences between mannan from C. auris and C. albicans as well

357 with the unstimulated cells have been observed (Extended Data Fig. 5b). 
359 Unique structure of $C$. auris mannans

360 Nuclear magnetic resonance (NMR) spectroscopy analyses of respective cell wall components for both

361 Candida species revealed no structurally unique features in $\beta$-glucans isolated from C. auris. However,

362 the distance between side-chain branching points (average number of $\beta$-linked glucosyl repeat units),

363 was larger for $C$. auris $\beta$-glucans than for $C$. albicans $\beta$-glucans (Supplementary table 3 ). In contrast,

364 these side chains were much shorter for $C$. auris than for $C$. albicans $\beta$-glucans. For $C$. auris mannans,

365 the acid stable portion was similar across all clinical isolates, revealing long side chains with varying

366 lengths containing linked $\alpha-1,2$-mannose, $\alpha-1,3$-mannose and $\beta$-1,2-mannose in varying amounts

367 (Figure 4). Strikingly, the acid labile portion of $C$. auris mannans revealed two distinct M- $\alpha-1-$

368 phosphate side chains (Figure 4), marking a unique structural feature that has not been observed in

369 the fungal kingdom before. Subsequent multi-detector gel permeation chromatography highlighted $C$.

370 auris mannans as extremely small biopolymers with a molecular weight (MW) ranging from 6.1 to 16.1

$371 \times 10^{3} \mathrm{Da}$ among the clinical isolates. This represents a major difference from C. albicans mannan,

372 which has a MW of $500 \times 10^{3} \mathrm{D}$ (Supplementary table 3).

373 We assessed the effects of these distinct and unique structural features of $C$. auris mannans

374 on their binding capacity with rhDectin-2 and rhMannose receptors, compared to C. albicans

375 mannans. Variability in binding affinities was observed amongst mannans from the different clinical $C$.

376 auris isolates, ranging from an equilibrium dissociation constant $\left(\mathrm{K}_{\mathrm{D}}\right)$ of 1.0 to $6.0 \mu \mathrm{M}$ for rhDectin-2

377 and 2.1 to $6.3 \mu \mathrm{M}$ for rhMannose. Although differentially recognized by both receptors, their overall

378 binding affinities were an order of magnitude lower than those observed for C. albicans mannans

379 (Supplementary table 3). Moreover, of the examined structural features, the affinity of $C$. auris

380 mannans for the rhDectin-2 receptor was solely associated with a higher MW $\left(r^{2}=0.4488, p=0.034\right)$. A

381 slightly lower association was observed for rhMannose $(p=0.096)$ yet displaying a similar trend.

382 Collectively, our data suggest that the double $M-\alpha-1$-phosphate side chains and small molecular size, 
384 the decreased recognition efficiency by two important anti-fungal recognition receptors.

385

386

387

388

389

390

391

392

393

394

395

396

397

398

399

400

401

402

403

404

405

406

407

The CLRs complement receptor 3 (CR3) and MMR contribute to the $C$. auris-induced cytokine production

Next, we investigated the downstream effects of the reduced binding affinity of $C$. auris mannans on intracellular signaling pathways and the subsequent activation of the immune system. The spleen tyrosine kinase, Syk, is an important mediator downstream of several $\mathrm{CLRs}^{38}$. CLR signaling from Dectin-1 and other lectins also involves the serine/threonine-protein kinase Raf- ${ }^{39}$. Inhibition of Syk and Raf-1 decreased TNF $\alpha$, IL-6, and IL-1 $\beta$ production in response to C. auris stimulation (Figure 5a), indicating the involvement of these two signaling pathways in cytokine production.

Due to the importance of Syk and Raf-1 pathways in CLR pathway mediation, we subsequently hypothesized a role for these receptors in C. auris recognition. Therefore, we pre-incubated PBMCs with neutralizing antibodies against important Candida CLRs (e.g., Dectin-1, Dectin-2, Mincle, DC-SIGN, MMR, CR3, and their control isotypes), one hour prior to stimulation with live C. albicans or C. auris. As expected, blocking Dectin-1 significantly decreased TNFa production upon C. albicans stimulation but, surprisingly, increased TNFa upon C. auris stimulation. (Figure 5b). We observed a significant reduction in C. auris-induced IL-6 and IL-1Ra production upon blocking of MMR (Figure 5b). Interestingly, neutralization of Dectin-1 and DC-SIGN led to a significant increase of IL-6 induced by C. auris compared to $\lg G 2 \mathrm{~b}$ isotype control. Moreover, blockade of CR3 led to a significant reduction in $\mathrm{IL}-1 \beta$ production and an increase in IL-1Ra production (Figure $5 \mathrm{~b}$ ). We conclude that CR3 and MMR signaling promotes cytokine production in response to C. auris, while blocking Dectin-1 functionality perturbs this cytokine production.

408 To evaluate the virulence of $C$. auris in vivo, immunocompetent C57BL/6J mice were injected 
intravenously (i.v.) with either $10^{7}$ Colony Forming Unit (CFU) of C. auris 10051895 ( $n=10$ ) or C.

410 albicans 10061110 ( $n=11$ ). Their survival was monitored over the course of 14 days. Significantly more

411 immunocompetent mice survived infection with C. auris than with C. albicans ( $3 / 11$ deaths for $C$. auris

412 and $11 / 11$ deaths for $C$. albicans over 14 days; Chi square=21.42; $p<0.0001$, Mantel-Cox test) (Figure

413 6a). In order to evaluate the differential organ invasion capacity between C. auris and C. albicans

414 infection, we injected intravenously either $10^{6}$ CFU of $C$. auris or C. albicans and sacrificed mice at day

4153 ( $n=5$ with C. albicans, $n=5$ with C. auris) and day 7 ( $n=4$ with C. albicans, $n=5$ with C. auris) for CFU

416 counting both in liver and kidneys. Although after day 3 there weren't any significant differences, a

417 significantly lower fungal burden has been found at day 7 in the kidneys of $C$. auris infected mice

418 compared to C. albicans-infected (Figure 6b). To confirm whether the ex vivo higher pro-inflammatory

419 response towards C. auris found in human PBMCs holds in vivo, we measured myeloperoxidase (MPO)

420 in organs and several cytokines both in plasma and organs of mice infected with either $10^{6} \mathrm{CFU}$ of $\mathrm{C}$.

421 albicans or $C$. auris. Except for a significantly lower Keratinocyte chemoattractant (KC) at day 7 (Figure

$4226 \mathrm{c}$ ), no significant differences between cytokine levels have been found between $C$. auris and $C$.

423 albicans-infected mice (Figure 6d and Extended Data Fig 6a-e). To understand the inflammatory

424 cytokine induction after the same load of Candida CFU, we normalized the inflammatory cytokine

425 production with the actual remaining CFU in organs by calculating the ratio of the mean MPO/CFU and

$426 \mathrm{KC}$ /CFU. MPO and KC production per remaining $C$. auris CFU were higher than the remaining $C$.

427 albicans CFU count (Figure 6f), supporting our in vitro findings in human PBMCs. In conclusion,

428 improved survival and better clearance of invasive $C$. auris infection as compared to $C$. albicans is

429 ensured by an adequate immune response in immunocompetent mice. 
433 In the current study, we investigated the transcriptional and functional responses of human PBMCs

434 and murine BMDMs to the rapidly emerging fungal pathogen C. auris. C. auris-induced host responses

435 were compared to those elicited by C. albicans, as this species remains the most frequent cause of

436 nosocomial fungal infections in humans to date ${ }^{33}$. A broad assessment of various clinical strains, as

437 well as further verification amongst the five $C$. auris clades, revealed that, with the exception of clade

$438 \mathrm{~V}, \mathrm{C}$. auris induces a stronger immune response than C. albicans in vitro. Functional and structural

439 assessment of $\beta$-glucans and mannans highlighted the presence of small and structurally unique $C$.

440 auris mannans, which were crucial for immune recognition. Compared to C. albicans, C. auris isolates

441 examined in this study were more efficiently phagocytosed by immune cells, induced lower levels of

442 macrophage lysis, and displayed lower virulence in a murine model of disseminated infection.

443 C. auris induced robust transcriptional changes in human PBMCs. These included both

444 common pathways induced by C. albicans as well, but also more robust specific IFN-dependent

445 transcriptional programs and explicit cytokine responses. This conclusion is supported by a recent

446 study by Mora-Montes and colleagues ${ }^{31}$. Secondly, C. auris appear to induce stimulation of immune

447 cells by sequential engagement of different components of the cell wall. The early (4 hour) responses

448 are mainly induced by $\beta$-glucans, and this initial phase of the response is largely similar to that induced

449 by $C$. albicans. This is probably explained by the similar structure of $C$. auris and $C$. albicans $\beta$-glucans.

450 In contrast, the late transcriptomic responses (24 hours) induced in PBMCs by C. auris display

451 significant differences and broader upregulation of immune genes compared with those induced by $C$.

452 albicans. These late responses are mainly mediated by C. auris mannoproteins with a specific

453 structure that includes a unique $\mathrm{M}-\alpha-1$-phosphate side chain in the acid labile portion of $\mathrm{C}$. auris

454 mannans, which has not been observed in the fungal kingdom to date. In line with the results of Yan et

455 al. reporting that $C$. auris mannans strongly bind to serum IgG and mannose-binding lectin (MBL) ${ }^{40}$, 
we showed that opsonization by human serum was necessary for $C$. auris mannan-induced cytokine

457 production.

458 By comparing C. albicans induced cytokine production with the different $C$. auris clades, we 459 observed variability that is probably linked to their distinct phenotypic, epidemiological, and drug

460 resistance properties. In particular, clade $V$ was the least immunogenic, while clades I and IV were the

461 strongest inducers of cytokine production. The amount of cytokines induced by the various strains

462 might be correlated to clade-specific characteristics which, in turn, might influence the level of

463 colonization/persistence in the host. When a healthy host encounters $C$. auris from clade I or IV, it

464 responds with a prompt high pro-inflammatory cytokine response which is protective. Although it is

465 also linked with invasive infections, clade III isolates show a lower in vitro cytokine production

466 compared to isolates from other clades. This may be linked to their tendency to form aggregates,

467 which might make innate immune recognition challenging. Finally, the fact that Clade II has a relatively

468 lower cytokine production, despite a higher phagocytosis rate as compared with C. albicans. This

469 might be related to its simpler mannan structure compared to isolates from Clade I and Clade IV

470 (Figure 4).

471 An important question concerns the PRRs responsible for the recognition of $C$. auris. Our

472 experiments using neutralizing antibodies revealed a significant role for the CLRs, especially MMR and

473 CR3, in the induction of cytokines by C. auris. The role of these receptors in the recognition of

474 mannans is well known ${ }^{41}$. However, our binding affinity data show that $r-M M R$ binds $C$. auris mannans

475 with a low affinity, and the in vitro neutralization of MMR led to only partial loss of cytokine

476 production, arguing that additional mannan-recognizing receptors contribute to anti-C. auris host

477 defense. On the other hand, the blocking of Dectin-1 significantly increased TNFa and IL-6 upon C.

478 auris stimulation for 24 hours. This interesting observation could be explained by differences in

479 relative $\beta$-glucan abundance. However, recently Navarro-Arias et al. ${ }^{31}$ quantified the abundance of

480 different cell wall components and found that total $\beta$-glucan and mannans in C. auris were

481 comparable to $C$. albicans. We suggest that this phenomenon might be due to a combination of two 
482 factors: i) the different exposure of $C$. auris $\beta$-glucan as compared to $C$. albicans (Extended data Fig.

4834 4); ii) the differential and variable cell wall adaptation of $C$. auris strains during the interaction with

484 the host which determines Dectin-1 dependence of $C$. auris host response. Such phenomenon occurs

485 in vivo during C. albicans infection in a strain-specific way and the differences in the levels of cell-wall

486 chitin influence the role of Dectin- $1^{42}$. Interestingly, Navarro-Arias et al. have reported significantly

487 more cell wall chitin in C. auris compared to C. albicans ${ }^{31}$. Since high chitin levels reduce the

488 dependence on Dectin-1 recognition, we speculate that differences in C. auris cell wall adaptation to

489 the host, variations in chitin content (higher chitin in C. auris) and differences in cell wall structure

490 (less exposure of beta glucan in C. auris and structurally unique mannoproteins) might provide an

491 explanation, at least in part, for the C. auris Dectin-1 (in)dependency.

492

Cytokine induction is important upon pathogen recognition, but the induction of phagocytosis

493 is also crucial ${ }^{43}$. We observed a higher phagocytic index for $C$. auris compared to C. albicans. This is

494 likely due to a better recognition of $C$. auris mannans by immune cells, as cell wall glycosylation is

495 critically important for the recognition and ingestion of $C$. albicans by macrophages ${ }^{44}$. Therefore, to

496 shed more light on these processes, future investigations might examine the phagocytosis dynamics of

497 C. auris mutant strains that are defective in their cell wall architecture. Interestingly, when the fate of

498 the fungus was assessed through video time-lapse microscopy, it was also clear that the continued cell

499 division of $C$. auris leads to altered $\mathrm{MOI}$ that are greater than $C$. albicans and this may also contribute

500 to the stronger stimulation of inflammation. However, this did not result in the death of the

501 phagocytes, most likely due to the lack of hyphae formation by engulfed $C$. auris cells. In line with

502 previous studies pointing to glucose competition as the main effector mechanism through which $C$.

503 albicans induce macrophage death ${ }^{45}$, a clear enrichment of glucose, fructose and mannose

504 metabolism pathways in the host were observed unique for 24-hour C. albicans exposure (as seen in

505 Figure $1 \mathrm{~A}$ and Supplementary Table 2). Conversely, the respective absence of the induction of such a

506 metabolic shift by $C$. auris suggests that this may be a likely mechanism to explain the lower

507 macrophage lysis induced by phagocytosed C. auris, and subsequently may explain the lower 
virulence. In addition, this could represent an important difference of the paths followed by different

509 Candida species as survival mechanisms.

$510 \quad$ The stronger induction of cytokines and lower macrophage lysis after 3 hours of phagocytosis

511 might have been expected to lead to lower virulence of $C$. auris in vivo compared with C. albicans. In

512 line with this hypothesis, experiments in a model of murine disseminated candidiasis demonstrate

513 that $C$. auris is less virulent compared to $C$. albicans, a conclusion supported by recent additional

514 studies ${ }^{46,47}$. Neutrophils are considered one of the most important host immune response to fungi

515 through phagocytosis and intracellular killing, or by releasing NETs ${ }^{48}$. In a recent study ${ }^{30}$, human

516 neutrophils were poorly recruited to sites of $C$. auris infection, were less able to kill $C$. auris compared

517 to $C$. albicans and failed to form NETs in response $C$. auris. However, neutrophils are important

518 contributors to the host defense against Candida species ${ }^{49}$. Our in vivo results show that MPO

519 production in C. auris-infected mice is similar to C. albicans, indicating that neutrophil activation is

520 comparable. In addition, the comparable innate and adaptive cytokine production in mice infected

521 with C. albicans and C. auris, as well as the similar or even better organ clearance, suggest that the

522 immune response toward C. auris is fully functional in an immunocompetent host. When the cytokine

523 levels were linked to the remaining organ CFU count by a cytokine/CFU ratio, we observed that there

524 was a higher cytokine production per singular $C$. auris CFU cultured from the organ, as compared to $C$.

525 albicans. This is in line with the potent pro-inflammatory response observed in human PBMCs

526 stimulated with C. auris. Future studies are warranted to dissect the relative importance of

527 neutrophils and macrophages in the host defense against $C$. auris.

528 In conclusion, we performed a first comprehensive assessment of the innate host defense

529 mechanisms against the rapidly emerging human pathogen $C$. auris. The overall conclusion is that the

530 host defense mechanisms induced by $C$. auris are generally classical antifungal mechanisms, but

531 important specific responses are also triggered by unique $C$. auris-specific mannoprotein structures.

532 The ensuing immune responses are effective and lead to an effective elimination of the fungus. Our

533 study argues that the intrinsic virulence of $C$. auris is not higher than other Candida species circulating 
534 in the patient population, but it is rather the infection control problem of this pathogen and its high

535 resistance to antifungal drugs that make it dangerous. The challenges that need to be pursued in the

536 coming years are to identify in even more detail the most effective components of the anti-C. auris

537 host defense, and to design and test novel host-directed therapies to enhance these pathways and

538 improve the outcome to the infection. In this respect, based on our results highlighting the peculiarity

539 of $C$. auris mannoprotein structures, one promising therapeutic possibility could be fosmanogepix

540 (APX001A), a novel agent that targets the fungal protein Gwt1 (glycosylphosphatidylinositol-anchored

541 wall transfer protein 1), thereby inhibiting the maturation and localization of GPI-anchored

542 mannoproteins in the cell wall ${ }^{20}$. Several studies reported higher survival rates and lower fungal

543 burden in C. auris infected mice treated with this novel drug ${ }^{20,50}$. One of the reasons for this higher

544 efficacy could be the crucial role of mannans for $C$. auris pathogenicity in the host. In addition to being

545 the first comprehensive study of the host immune response to $C$. auris, our data provide further

546 support from an immunological and microbiological perspective for the development of drugs

547 potentially targeting mannan synthesis as new and efficient anti-fungal drugs for $C$. auris.

548

549 
551 EXPERIMENTAL MODEL AND SUBJECT DETAILS

552

553 Ethics statement for ex vivo human PBMC stimulations

554

555 Ex vivo/in vitro human PBMC stimulations - Inclusion of healthy controls was approved by the local

556 institutional review board (CMO region Arnhem-Nijmegen, \#2299 2010/104) and conducted according

557 to the principles of the International Conference on Harmonization-Good Clinical Practice guidelines.

558 Buffy coats from healthy donors were obtained after written informed consent (Sanquin, Nijmegen,

559 the Netherlands).

560

561

Ethics statement for in vivo mice studies

562

All animal experiments were conducted in the unit of animals for medical scientific purposes of

563

University General Hospital 'Attikon' (Athens, Greece) according to EU Directive 2010/63/EU for

564 animal experiments and to the Greek law 2015/2001, which incorporates the Convention for the

565 Protection of Vertebrate Animals used for Experimental and Other Scientific Purposes of the Council

566 of Europe (code of the facility EL 25BIO014, approval no. 1853/2015). All experiments were licensed

567 from the Greek veterinary directorate under the protocol number 7467/24-12-2013. All animal

568 experiments were reported using the ARRIVE guidelines.

569

$570 \quad$ PBMCs isolation and stimulation

571 Venous blood from the antecubital vein of healthy volunteers was drawn in EDTA tubes after

572 obtaining written informed consent. PBMC isolation was performed as previously described ${ }^{51}$. Briefly,

573 the PBMC fraction was obtained using density centrifugation in Ficoll-Paque (Pharmacia Biotech,

574 Piscataway, USA). Cells were then washed twice in PBS and re-suspended in RPMI-1640+ medium

575 (RPMI1640 Dutch modification supplemented with $50 \mu \mathrm{g} / \mathrm{mL}$ gentamicin, $2 \mathrm{mM} \mathrm{L-glutamine}$ and $1 \mathrm{mM}$ 
pyruvate; Gibco, Invitrogen, Breda, The Netherlands). Afterwards, PBMCs were counted and re-

577 suspended in a concentration of $5 \times 10^{6}$ cells $/ \mathrm{mL}$. $5 \times 10^{5}$ PBMCs were added in $100 \mu \mathrm{L}$ to round-bottom

578 96-well plates (Greiner, Alphen Aan Den Rijn, NL) and incubated with $50 \mu \mathrm{L}$ of stimulus (RPMI, live, $4 \%$

579 paraformaldehyde (PFA) or heat killed Candida albicans yeast $1 \times 10^{6} / \mathrm{mL}$ or Candida auris $1 \times 10^{6} / \mathrm{mL}$;

$580100 \mu \mathrm{g} / \mathrm{mL}$ purified C. albicans or C. auris mannan; $10 \mu \mathrm{g} / \mathrm{mL}$ purified C. albicans or C. auris $\beta$-glucan)

581 and $50 \mu \mathrm{L}$ of eventual inhibitor or medium with or without $10 \%$ human serum. Serum was either

582 complement active, if not otherwise indicated, or heat inactivated by incubation for 30 minutes at

$58356^{\circ} \mathrm{C}$ in a water bath according to a commonly used protocol. After 1 hour of pre-incubation with

584 inhibitor or medium, stimuli or medium was added. In detail, for receptor blockade experiments,

585 before stimulation with C. albicans or C. auris, PBMCs were pre-incubated for 1 hour with 5

$586 \mu \mathrm{g} / \mathrm{mL}$ anti-DC SIGN antibody $10 \mu \mathrm{g} / \mathrm{mL}$, anti-Dectin-1, $10 \mu \mathrm{g}$ anti Mincle and $10 \mu \mathrm{g} / \mathrm{mL}$ control

587 IgG2b; $10 \mu \mathrm{g} / \mathrm{mL}$ anti-Dectin-2 antibody and $10 \mu \mathrm{g} / \mathrm{mL}$ of its control IgG1]); $10 \mu \mathrm{g} / \mathrm{mL}$ anti-CR3

588 antibody and control IgG (R\&D), $10 \mu \mathrm{g} / \mathrm{mL}$ MR-blocking antibody and $10 \mu \mathrm{g} / \mathrm{mL}$ Goat IgG

589 isotype control. After 1 hour, cells were stimulated with $10^{6}$ heat-killed C. albicans and C.

590 auris. For the intracellular pathways blockade experiment 50 nM Syk inhibitor, $1 \mu \mathrm{M}$ Raf-

591 inhibitor or the same concentration of vehicle (DMSO) has been used. Concentrations of

592 inhibitors were selected as being the highest non-cytotoxic concentrations. All supernatants

593 were stored at $-20^{\circ} \mathrm{C}$ until analyzed.

\begin{tabular}{|l|l|l|}
\hline \multicolumn{2}{|l|}{ Antibodies } & Source \\
\hline Product & $\begin{array}{l}\text { Article } \\
\text { Number }\end{array}$ \\
\hline & $\begin{array}{l}\text { Fisher } \\
\text { Scientific }\end{array}$ & MA1-25615 \\
\hline $\begin{array}{l}\text { DC-SIGN Monoclonal Antibody (clone 120507) } \\
\text { 259931) }\end{array}$ & $\begin{array}{l}\text { Bio- } \\
\text { Techne/R\&D }\end{array}$ & MAB1859 \\
\hline Anti-hMincle-IgG & Invivogen & mabg-hmcl \\
\hline & $\begin{array}{l}\text { Bio- } \\
\text { Techne/R\&D }\end{array}$ & MAB004 \\
\hline Mouse IgG2B Isotype Control & $\begin{array}{l}\text { Bio- } \\
\text { Techne/R\&D }\end{array}$ & MAB3114 \\
\hline
\end{tabular}




\begin{tabular}{|c|c|c|}
\hline IgG1 Isotype Control & $\begin{array}{l}\text { Bio- } \\
\text { Techne/R\&D }\end{array}$ & MAB002 \\
\hline Human MMR/CD206 Antibody & $\begin{array}{l}\text { Bio- } \\
\text { Techne/R\&D }\end{array}$ & AF2534 \\
\hline $\begin{array}{l}\text { anti-hIntegrin beta2 - hIntegrin b2 Affinity Purified Goat } \\
\text { IgG }\end{array}$ & $\begin{array}{l}\text { Bio- } \\
\text { Techne/R\&D }\end{array}$ & AF1730 \\
\hline
\end{tabular}

594

595 Cytokine and lactate measurements

596 All cytokine levels were measured in the cell culture supernatants using commercially

597 available ELISA assays according to the protocol supplied by the manufacturer. For human

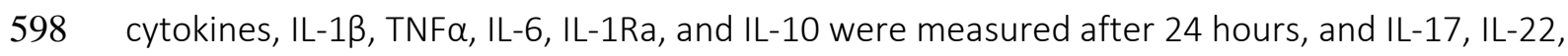

599 and IFN- $\gamma$ were measured after 7 days of stimulation. For mouse cytokines, KC, IL-13, IL-6, IL-

60010, IL-17, and IFN- $\gamma$ were measured after 3 and 7 days. Lactate was measured by a Lactate

601 Fluorometric Assay Kit (Biovision, Milpitas, USA).

602

\section{ELISA assays}

\begin{tabular}{|l|l|l|}
\hline Product & Source & $\begin{array}{l}\text { Article } \\
\text { Number }\end{array}$ \\
\hline Human IL-1 $\beta$ ELISA & R\&D Systems & DY201 \\
\hline Human TNF $\alpha$ ELISA & R\&D Systems & DY210 \\
\hline Human IL-17 ELISA & R\&D Systems & D1700 \\
\hline Human IL-22 ELISA & R\&D Systems & D2200 \\
\hline Human IL-6 ELISA & R\&D Systems & DY206 \\
\hline Human IL-8 ELISA & Sanquin & M1918 \\
\hline Human IL-10 ELISA & R\&D Systems & D1000B \\
\hline Human IFN- - ELISA & Sanquin & M1933 \\
\hline Mouse KC ELISA & R\&D Systems & MKC00B \\
\hline Mouse IL-1 $\beta$ ELISA & R\&D Systems & MLB00C \\
\hline Mouse IL-6 ELISA & R\&D Systems & M6000B \\
\hline Mouse IL-10 ELISA & R\&D Systems & M1000B \\
\hline Mouse IL-17 ELISA & R\&D Systems & M1700 \\
\hline Mouse IFN- - ELISA & R\&D Systems & MIF00 \\
\hline
\end{tabular}

603

604 Cytotoxicity assay 
607 of live Candida auris $1 \times 10^{6 /} \mathrm{mL}$ ) for 24 hours. Cell viability was assessed using CytoTox 96 non-

608 radioactive cytotoxicity assay (Promega, Leiden, the Netherlands) according to the

609 manufacturers' instructions. Released lactate dehydrogenase (LDH), a stable cytosolic enzyme

610 released upon cell lysis, was measured in the supernatant with a 30-minute coupled

611 enzymatic assay. The color intensity, as a result of the conversion of tetrazolium salt (INT) into

612 ref formazan, is proportional to the number of lysed cells. As positive control, cells were lysed 613 with $0.5 \%$ Triton-X reflecting maximal LDH release.

614

615 Candida strains

616 C. auris strains from five clades have been used (clade I, South Asia; clade II, East Asia; clade

617 III, South Africa; clade IV, South America; clade V, Iran). C. albicans and C. auris strains were

618 prepared by growing cells for $24 \mathrm{~h}$ in either a Sabouraud broth or on Sabouraud plates at $30^{\circ} \mathrm{C}$.

619 Unless otherwise indicated, experiments were performed using C. albicans CWZ10061110, C.

620 auris KCTC17810 reference, C. auris CWZ10051893 and C. auris CWZ10051895. Stimulations

621 were performed using either live, heat-killed (12 hours at $56^{\circ} \mathrm{C}$ ) or $4 \%$ PFA-killed

622 microorganisms. Heat killing caused the disruption of the outer layer of the Candida cell wall

$623{ }^{34}$ in turn enhancing $\beta$-glucan exposure ${ }^{35,36}$. While Candida killing via heat treatment disrupts

624 the outer layer causing exposure of $\beta$-glucan in the cell wall, with PFA fixation and thimerosal

625 fixation the cell wall structure remains intact ${ }^{34,52,53}$.

\begin{tabular}{|l|l|l|}
\hline Fungal Strains & from & name(s) \\
\hline C. albicans & ATCC & ATCC MYA-3573/UC820 \\
\hline C. albicans & ATCC & SC5314 / ATCC MYA-2876 \\
\hline
\end{tabular}




\begin{tabular}{|l|l|l|} 
C. albicans & $\begin{array}{l}\text { J. Meis (Clinical blood } \\
\text { isolate) }\end{array}$ & CWZ 10061110 \\
\hline $\begin{array}{l}\text { C. } \text { albicans } \\
\text { C. } \text { auris (Clade I) }\end{array}$ & $\begin{array}{l}\text { A. Chowinical blood } \\
\text { isolate) }\end{array}$ & CWZ 10070679 \\
\hline C. auris (Clade I) & A. Chowdhary & CWZ 10051893 (Clade I) \\
\hline $\begin{array}{l}\text { C. } \text { auris (Clade } \\
\text { II) }\end{array}$ & J. Meis & CWZ 10051895 (Clade I) \\
\hline $\begin{array}{l}\text { C. } \text { auris (Clade } \\
\text { III) }\end{array}$ & J. Meis & KCTC17810 (Clade II) \\
\hline $\begin{array}{l}\text { C. } \text { auris (Clade } \\
\text { IV) }\end{array}$ & J. Meis & CDC AR 383/B11221 (Clade III) \\
\hline $\begin{array}{l}\text { C. } \text { auris (Clade } \\
\text { V) }\end{array}$ & J. Meis & CWZ 10051522 (Clade IV) \\
\hline
\end{tabular}

626

627

628

$629 \quad \beta$-glucan and mannan isolation from $C$. auris strains

630 For the cell wall experiments, a total of 8 different $C$. auris clinical strains were used for

631 glucan and mannan extraction, originating from three different clades (I, II and IV). Isolates

632 were grown in $25 \mathrm{~mL}$ YPD (1\% yeast extract, $2 \%$ dextrose, $2 \%$ peptone) at $30^{\circ} \mathrm{C}$ for 48 hours

633 before parallel isolation of respective cell wall components under identical conditions. Aiding

$634 \beta$-glucan and mannan collection, cell walls were disrupted by 3 cycles of freeze/thawing (-

$63520^{\circ} \mathrm{C}$ ) and consecutive harvesting of cell pellets. Of each culture a small aliquot was plated on

636 YPD media to exclude the presence of viable cells, after which the cell pellets were suspended

637 in $10 \mathrm{~mL}$ of $0.75 \mathrm{~N} \mathrm{NaOH}$ to a final concentration of $\sim 3 \mathrm{mg} / \mathrm{mL}$. Suspensions were heated to

$638105^{\circ} \mathrm{C}$ for 15 minutes, cooled and separated by centrifugation (10 minutes $\times 863 \mathrm{~g}$ ). The

639 supernatant, containing the mannans, were harvested and dialyzed against 300 volumes of 18

640 MOhm water (2000 MWCO). After subsequent harvesting, the $\mathrm{pH}$ was confirmed to neutral,

641 followed by freezing and lyophilization to dryness. For $\beta$-glucans, the centrifuged extract was 
642 transferred, treated with $10 \mathrm{~mL}$ of $1.0 \mathrm{~N} \mathrm{H}_{3} \mathrm{PO}_{4}$ and heated $105^{\circ} \mathrm{C}$ for 15 minutes. After cooling

643 down, pellets were collected by centrifugation (10 minutes $\times 863 \mathrm{~g}$ ) and extracted once more

644 with $10 \mathrm{~mL} 100 \%$ ethanol containing $1 \%(\mathrm{v} / \mathrm{v}) \mathrm{H}_{3} \mathrm{PO}_{4}$ at $90^{\circ} \mathrm{C}$ for 15 minutes. $\beta$-glucans were

645 harvested by centrifugation and purified by washing $3 \mathrm{x}$ with $18 \mathrm{MOhm}$ water, after which the

646 final pellet was frozen and lyophilized to dryness.

647

648 C. albicans mannan isolation

649 Mannan isolation was performed using a modification of the Fehling method as previously

650 described ${ }^{54}$. Briefly, yeast cells, grown $\mathrm{O} / \mathrm{N}$ at $30^{\circ} \mathrm{C}$, were delipidated by suspending cell

651 pellets in $100 \mathrm{~mL}$ of acetone and incubation for 20 minutes. The supernatant was decanted

652 after 5-minute centrifugation at $500 \mathrm{rpm}$ and pellets were air-dried for 30 minutes.

653 Facilitating mannan extraction, pellets were suspended in $200 \mathrm{~mL}$ of distilled $\mathrm{H}_{2} \mathrm{O}\left(\mathrm{dH} \mathrm{H}_{2} \mathrm{O}\right)$ and

654 supplemented with an equivalent amount of glass beads $0.5 \mu$ to pellet weight and followed

655 by bead beating ( $3 \times 30$ second pulses). Cell extracts were autoclaved for two hours and

656 subsequently centrifuged for 5 minutes at 5,000 rpm. Respective supernatant was split into

657 two; one half was left untreated while the other was treated with $500 \mathrm{mg}$ of pronase for 16

658 hours at $37^{\circ} \mathrm{C}$ to abolish glycosidic activity. Ensuring the suppression of bacterial growth

659 during pronase treatment sodium azide was added at a final concentration of $50 \mathrm{nM}$. The

660 pronase-treated and untreated samples were 1:1 diluted with freshly prepared Fehling

661 solution and allowed to mix for 1 hour at RT, followed by a 20-minutes incubation allowing

662 precipitation of the mannan-copper complexes. After decantation, complexes were treated

663 with $5 \mathrm{~mL} \mathrm{HCl}$. When dissolved, $100 \mathrm{~mL}$ of a 1:8 mixture of glacial acetic acid:methanol

664 (GAE:MEOH) was added for mannan precipitation. After 4 hours, samples were washed

665 repeatedly with GAE:MEOH until the remaining precipitate appeared colorless (lack of any 
666 blue/green) and followed by 3x methanol washes. The final precipitate was dissolved in 100

$667 \mathrm{~mL}$ of $\mathrm{dH}_{2} \mathrm{O}$ and dialyzed against 300 volumes of $\mathrm{dH}_{2} \mathrm{O}$ over 48 hours and for 12 hours against

668200 volumes of ultrapure $\mathrm{dH}_{2} \mathrm{O}$ to remove any salts, acid, methanol and other low molecular

669 weight contaminants remaining from the extraction protocol. Dialyzed mannans were frozen

670 and lyophilized for $48-72$ hours and stored at $-20^{\circ} \mathrm{C}$.

\begin{tabular}{|c|c|c|}
\hline \multicolumn{3}{|l|}{ Cell wall components } \\
\hline Name & from & Fungal strains \\
\hline C. auris mannans 1 & David Williams & KCTC17810 (Clade II) \\
\hline C. auris mannans 2 & David Williams & CWZ 10031160 (2012) (Clade I) \\
\hline C. auris mannans 3 & David Williams & CWZ 10031163 (2012) (Clade I) \\
\hline C. auris mannans 4 & David Williams & CWZ 10051256 (2013) (Clade I) \\
\hline C. auris mannans 5 & David Williams & CWZ 10051263 (2013) (Clade I) \\
\hline C. auris mannans 6 & David Williams & CWZ 10051522 (2014) (Clade IV) \\
\hline C. auris mannans 7 & David Williams & CWZ 10051244 (2014) (Clade I) \\
\hline C. auris mannans 8 & David Williams & CWZ 10051252 (2014) (Clade I) \\
\hline C. albicans mannans & David Williams & SC5314 \\
\hline C. auris 6 -glucans 1 & David Williams & KCTC17810 (Clade II) \\
\hline C. auris 6 -glucans 2 & David Williams & CWZ 10031160 (2012) (Clade I) \\
\hline C. auris 6 glucans 3 & David Williams & CWZ 10031163 (2012) (Clade I) \\
\hline C. auris 8 -glucans 4 & David Williams & CWZ 10051256 (2013) (Clade I) \\
\hline C. auris 6 glucans 5 & David Williams & CWZ10051263 (2013) (Clade I) \\
\hline C. auris 6 glucans 6 & David Williams & CWZ10051522 (2014) (Clade IV) \\
\hline C. auris 6 glucans 7 & David Williams & CWZ 10051244 (2014) (Clade I) \\
\hline C. auris 6 glucans 8 & David Williams & CWZ 10051252 (2014) (Clade I) \\
\hline C. albicans $B$ glucans & David Williams & SC5314 \\
\hline
\end{tabular}

671

672 RNA purification

673 PBMCs from three healthy donors, with a concentration of $5 \times 10^{6}$ cells $/ \mathrm{mL}$, were stimulated

674 in flat-bottom 12-well plates (Corning, NY, USA) with freshly counted live C. albicans $\left(1 \times 10^{6} / \mathrm{mL}\right)$

675 and C. auris $\left(1 \times 10^{6} / \mathrm{mL}\right)$, and purified cell wall components $\beta$-glucans $(10 \mathrm{\mu g} / \mathrm{mL})$ and mannans

$676(10 \mu \mathrm{g} / \mathrm{mL})$ isolated from both Candida species as described above. Of note, the time between

677 Candida cell count and experiment start did not exceed 15 minutes. PBMCs were cultured in

678 the presence of $10 \%$ human pooled serum. At 4 and 24 hours cells were lysed with RLT 
679 buffer. Prior to subjection to the RNeasy Mini Kit (Qiagen), lysates were homogenized using a

$6801 \mathrm{~mL}$ syringe with a $0.8 \times 15 \mathrm{~mm}$ needle. RNA was subsequently extracted following

681 manufacturers' protocol, including an on-column DNAse digestion using the RNAse-Free

682 DNAse set (Qiagen). Quantification and quality assessment of extracted RNA was performed

683 using the Qubit RNA HS assay (Thermo Fisher Scientific) and Agilent 2200 TapeStation (RNA

684 HS Screentape, Agilent), respectively. The majority of samples subjected to quality

685 assessment revealed an RNA integrity number $\left(\mathrm{RIN}^{\mathrm{e}}\right)$ of $\geq 8$.

\section{QuantSeq 3' mRNA sequencing}

687 Libraries were generated from the extracted RNA using the QuantSeq 3' mRNA-Seq Library

688 Prep Kit-FWD from Lexogen (Lexogen, Vienna, Austria) in accordance to the manufacturers'

689 protocol. Three separate preparations were performed, split by PBMC donor, in turn limiting

690 the number of samples to 14 to 18 samples per prep. RNA input was normalized to $100 \mathrm{ng}$ for

691 donor A, and to 250 ng for donors B and C. An aliquot (1:10) of double stranded cDNA

692 libraries was used for quantitative PCR, in turn indicating $17-18$ cycles as optimal for

693 endpoint PCR (17- donor B; 18 - donors A, C). Accurate quantification and assessment of

694 quality of the generated libraries was performed using Qubit dsDNA HS assay (Thermo Fisher

695 Scientific, Waltham, USA) and Agilent 2200 TapeStation (HS-D1000 ScreenTape, Agilent, Santa

696 Clara, USA). The cDNA concentration and average fragment size were used to determine the

697 molar concentration of the individual libraries. Consequently, libraries were pooled equimolar

698 to $100 \mathrm{fmol}$. After a final dilution of the pool to a concentration of $4 \mathrm{nM}$, the libraries were

699 sequenced on a NextSeq 500 instrument (Illumina, San Diego, USA), with 75 cycle (i.e. 75bp

700 single-end sequence reads), high output kit with a $1.1 \mathrm{pM}$ final loading concentration. 
702 Quality of the acquired sequencing data was controlled using FastQC tool v0.11.5 (Babraham

703 Bioinformatics) and subsequently followed by the removal of adapter sequences and poly(A)

704 tails with Trim Galore! v.0.4.4_dev (Babraham Bioinformatics) and Cutadapt v1.18 ${ }^{55}$. On

705 average $\sim 6$ million reads per individual library were retrieved. Filtered and trimmed reads

706 were mapped to the human reference genome (hg38/GRCh38) using the STAR aligner v2.6.0a

707 (Supplementary Table 4$)^{56}$. Less than $1 \%$ of all reads were comprised of overrepresented

708 sequences and were uniquely mapped with a median of 4 million reads (74.1\%). After

709 generating gene level count data using the HTSeq-count tool v0.11.0 ${ }^{57}$, an additional filtering

710 step was performed ensuring the exclusion of several non-coding RNAs, i.e. mtRNA, lincRNA,

711 snRNA, tRNA, miscRNA and snoRNA, in our dataset. Given the absence of sample replicates,

712 PBMC donors were considered biological replicates. Hence, in the differential gene expression

713 analysis using DESeq2 v1.22.0, including logFold Shrinkage and apeg/m ${ }^{58}$, the average PBMC

714 donor response to the different stimuli were compared to their control condition, RPMI.

715 Genes with a fold change of $\geq 2$ and a p-adjusted value $<0.01$ were considered differentially

716 expressed genes (DEG). Allowing identification of the main principle components

717 underpinning the majority of the variance, a PCA analysis was performed on DESeq2

718 normalized counts (normTransform).

719

720 Pathway enrichment analysis

721 In order to distinguish between the responses triggered by both Candida species, DEG were

722 compared between species for the analogous stimulations (live, mannan and $\beta$-glucan), and

723 corresponding time-points. In turn resulting a group of DEG that overlap between the two

724 species, and DEG that were uniquely attributed to either one of the Candida species. 
725 Overrepresentation analysis were performed on all groups per stimulation (and time-point)

726 using Consensus PathDB ${ }^{59}$, including pathways as defined by pathway databases Kyoto

727 Encyclopedia of Genes and Genomes KEGG ${ }^{60}$ and Reactome ${ }^{61}$. Minimum overlap in input

728 was set at 2, together with a p-value cut-off of 0.01 . For downstream analysis, pathways were

729 considered enriched with a corrected p-value <0.01 (indicated as 'q-value').

730

731 Structural characterization of mannans by NMR spectroscopy

732 To gain insight into the mannan structure of both Candida species, isolated mannans were

733 subjected to solution-state $1 \mathrm{D}^{31} \mathrm{P}$-coupled and ${ }^{31} \mathrm{P}$-decoupled ${ }^{1} \mathrm{H}$ NMR spectroscopy and $2 \mathrm{D}$

734 COSY NMR spectroscopy. Data acquisition and subsequent analysis were based on methods

735 described by Lowman et al. ${ }^{62}$. In short, ${ }^{1} \mathrm{H}$ NMR spectra were collected using a Bruker Avance

736 III $600 \mathrm{NMR}$ spectrometer operating at $331^{\circ} \mathrm{K}\left(58^{\circ} \mathrm{C}\right)$. Roughly $10 \mathrm{mg}$ of mannan was dissolved

737 in $600 \mathrm{~mL}$ of $\mathrm{dH}_{2} \mathrm{O}$. Chemical shift referencing was accomplished relative to TMSP at $0.0 \mathrm{ppm}$.

738 Proton 1D NMR spectra were collected with 2 dummy scans, 256 scans, 65,536 data points,

73920 ppm sweep width centered at $6.2 \mathrm{ppm}$, and 1 second pulse delay. For the $1 \mathrm{D}^{31} \mathrm{P}$

740 decoupled ${ }^{1} \mathrm{H}$ NMR experiment, spectra were collected at $333^{\circ} \mathrm{K}\left(60^{\circ} \mathrm{C}\right)$ with 2 dummy scans,

7411024 scans, 65,536 data points, 21 ppm sweep width centered at $6.2 \mathrm{ppm}$ and the ${ }^{31} \mathrm{P}$

742 decoupling pulse centered at $3.0 \mathrm{ppm}$. All 1D spectra were processed using exponential

743 apodization with $0.3 \mathrm{~Hz}$ line broadening. COSY spectra were collected using 2048 by 128 data

744 points, 8 dummy scans, 32 scans, and 6.0 ppm sweep width centered at $3.0 \mathrm{ppm}$ and

745 processed with sine apodization in both dimensions and zero-filled to 1024 data points in f1.

746 Processing was accomplished with the JEOL DELTA (version 5.0.4.4) and Bruker TopSpin

747 (version 4.0.6) software packages.

748 
750 To determine the MW of mannans, isolates from C. albicans and C. auris strains were

751 subjected to high performance gel permeation chromatography (GPC) as previously described

752 63. Using a Viscotek/Malvern GPC system, consisting of a GPCMax autoinjector fitted to a TDA

753305 detector (Viscotek/Malvern, Houston, TX). System calibration was achieved using

754 Malvern pullulan and dextran standards. Mannan isolates, ranging between 3 to $6 \mathrm{mg} / \mathrm{mL}$,

755 dissolved in mobile phase (50 mM of sodium nitrate, $\mathrm{pH} 7.3$ ) were subjected to a 60 -minute

756 incubation at $60^{\circ} \mathrm{C}$, followed by sterile filtration $(0.2 \mu \mathrm{m})$ and injected into the GPC (100-200

$757 \mu \mathrm{L})$. Samples were analyzed in duplicate or triplicate and data analysis performed with

758 Viscotek OmniSec software (version 4.7.0.406).

759

760 Binding interaction of mannans with PRRs rhDectin-2 and rhMannose

761 Assessment of mannan binding interactions to the recombinant Dectin-2 and Mannose

762 receptors (R\&D systems) were carried out on an Octet K2 BLI instrument (ForteBio) in 10X

763 Kinetics Buffer $\left(\mathrm{pH} \mathrm{7.4)}\right.$ at $30^{\circ} \mathrm{C}$ and 1000 rpm. Increasing concentration of the respective

764 ligands (3.125-400 $\mu \mathrm{g} / \mathrm{mL})$ were used to generate respective saturation curves, after which

765 the binding affinities were calculated for mannans isolated from C. albicans and C. auris

766 strains as previously described ${ }^{64}$. The Ni-NTA biosensor was subjected to a 3-minute

767 equilibration prior to 10 minutes of exposure $0.1 \mathrm{ug} / \mathrm{mL}$ HIS-tagged rhDectin-2 or rhMannose

768 receptor proteins and final a 10-minute dissociation in 10X kinetics buffer for measuring the

769 BLI signal, consistently 20 seconds after transferring. Subsequently followed by a series 8

770 similar 5-minute exposure to an increasing concentration (2-fold) of carbohydrate. To control

771 for receptor dissociation during the experiment, a parallel biosensor with the immobilized

772 receptors was placed in the 10X kinetics buffer without respective carbohydrate exposure. 
773 Data analysis was performed using the GraphPad Prism 7.0 software and the dissociation

774 constant $K_{D}$ is presented as mean value with a $95 \%$ confidence interval.

775

776 ROS assay

777 The induction of ROS was measured by oxidation of luminal (5-amino-2,3, dihydro-1,4-

778 phtalazinedione) and determined in an automated LB96V Microlumat plus luminometer (EG \& G

779 Berthold, Bad Wildbad, Germany). Briefly, PBMCs $\left(5 \times 10^{5}\right.$ per well $)$ or neutrophils $\left(2.5 \times 10^{5}\right)$ per well

780 were seeded into white 96-well plates and incubated in medium containing either RPMI, Zymosan

$781(100 \mu \mathrm{g} / \mathrm{mL})$, heat-killed opsonized C. albicans or C. auris yeast $\left(10^{7} \mathrm{CFU} / \mathrm{mL}\right) .20 \mu \mathrm{L}$ of $1 \mathrm{mM}$ Luminol

782 was added to each well in order to start the chemiluminescence reaction. Each measurement was

783 carried out at least in duplicate. Chemiluminescence was determined every 145 seconds at $37^{\circ} \mathrm{C}$ for 1

784 hour. Luminescence was expressed as relative light units (RLU) per second. The RLU/sec within the

785 area under the curve (AUC) were plotted against time and analyzed by using Graphpad Prism v.7.0.

786

$787 \quad$ FITC-labelling of Candida

788 To label cells with fluorescein isothiocyanate (FITC, CAS Number: $3326-32-7), 1 \times 10^{8} / \mathrm{mL}$ of

789 thimerosal fixed cells were sonicated and resuspended in $0.1 \mathrm{mg} / \mathrm{mL}$ of FITC in $0.1 \mathrm{M}$

790 carbonate-bicarbonate buffer $\left(\mathrm{pH}\right.$ 9.6). After incubation for 30 minutes on a tube roller at $4^{\circ} \mathrm{C}$

791 in the dark, unbound FITC was washed away by centrifugation at $3000 \mathrm{rpm} 4^{\circ} \mathrm{C}$ for 10 minutes

792 three times in PBS. Before use, cells were resuspended to a concentration of $1 \times 10^{7} / \mathrm{mL}$ in PBS,

793 aliquoted and stored in the dark at $-20^{\circ} \mathrm{C}$.

794

795 Phagocytosis assay in human cells

796 To test Candida strains uptake by human monocytes, $4 \times 10^{6}$ cells $/ \mathrm{mL}$ of thimerosal-killed

797 FITC-labelled C. albicans and C. auris were pre-opsonized with $20 \%$ human pooled serum for 1 
798 hour at $37^{\circ} \mathrm{C}, 5 \%$ and subsequently incubated with $2 \times 10^{5} \mathrm{PBMCs} /$ well (MOI 2:1,

799 fungal:human cells) for either 30 minutes or 2 hours at $37^{\circ} \mathrm{C},+5 \% \mathrm{CO}_{2}$. After the incubation

800 period, cells were gently washed with PBS (1\% BSA) and then stained in a total volume of 50

$801 \mu \mathrm{L}$ using CD14 monoclonal antibody (Mouse-anti-Human CD14 Pacific Blue, Beckman coulter,

802 clone RM052, dilution 1:20) for 30 minutes at $4^{\circ} \mathrm{C}$ on ice in the dark. Afterwards, cells were

803 washed, and the fluorescence signal of extracellular non-phagocytosed Candida was

804 quenched with 0.1\% Trypan blue solution (Sigma, St. Louis, USA; CAS Number 72-57-1). Cells

805 were subsequently measured on a CytoFLEX flow cytometer (Beckman coulter, Pasadena,

806 USA) and the data were analyzed using the Kaluza Analysis software version 2.1. To determine

807 the uptake of $C$. albicans and $C$. auris by human monocytes, the percentage of CD14 positive

808 cells which had phagocyted FITC positive Candida percentage of FITC-positive cells in the

809 CD14-positive population) was calculated. For the detailed gating strategy see Extended data

$810 \quad$ Fig. 7a.

811

812 Phagocytosis assay in BMDMs

813 Bone marrow was extracted from femurs and tibias of eight-week-old male C57BL/6 mice and

814 differentiated for 7 days with RPMI Medium 1640 Glutamax (Gibco) supplemented with 10\%

815 heat-inactivated foetal calf serum, 100 U/mL Penicillin/Streptomycin and 15\% L929 cell-

816 conditioned medium at $37^{\circ} \mathrm{C}$ with $5 \% \mathrm{CO}_{2}$. $\mathrm{BMDM}$ were added to 8 well $\mathrm{u}$-slide (ibidi,

817 Gräfelfing, Germany) at $0.5 \times 10^{5}$ cells per well to adhere overnight. C. albicans and C. auris

818 strains were prepared by growing cells for 24 hours in Sabouraud broth at $30^{\circ} \mathrm{C}$ and followed

819 by 3 washes in PBS. Fixed Candida cells were prepared by incubating the Sabouraud-grown

820 yeast overnight at room temperature in $50 \mathrm{Mm}$ thimerosal (Sigma, St. Louis, USA) followed by

8215 wash steps in PBS. Phagocytosis dynamics were determined following the addition of 3:1; 
822 yeast: BMDM. The intended Multiplicity of Infection (MOI) was calculated using cell count

823 (hemocytometer) and the actual $\mathrm{MOI}$ was observed from videos. Live imaging of macrophage

824 interactions with live or fixed C. albicans and C. auris were performed using a Nikon Ti Eclipse

825 microscope with objective 20x magnification set to acquire images at 1-minute intervals using

826 Volocity software (Version 6.3, PerkinElmer, Waltham, USA), with thanks to the University of

827 Aberdeen Microscopy Core Facility. Movies generated from 3 hour interactions were

828 analyzed to determine over time the proportion of macrophages phagocytosing yeast (\%

829 uptake), the number of yeast phagocytosed per 100 macrophages (phagocytic index), the

830 proportion of macrophage death after 3 hours (macrophage lysis) and the distribution of

831 yeast contained within individual macrophages. Experiments were performed on 3 occasions,

832 with a total of 9 movies generated per condition. Phagocytic index data are based on yeasts

833 fully inside macrophages. Yeasts adhering but not internalized would not be included in cell

834 counts for phagocytic index. Statistical analyses were performed by ANOVA using GraphPad

835 Prism (v 7.0).

836

837 Cell wall staining

838 Fixed Candida yeast were stained for exposed cell wall $\beta$-glucans using Fc-Dectin-1 (a gift from

839 Gordon Brown, University of Aberdeen) and secondary F(ab')2 anti-human IgG AlexaFluor 488

840 conjugate (Life Technologies). ConA-Texas Red conjugate (Life Technologies, Carlsbad, USA)

841 was used to detect cell wall mannans. Cells were counted, and $2.5 \times 10^{6}$ yeast were combined

842 with FACS wash (1\% bovine serum albumin and 5 mM EDTA in PBS) with either Fc-Dectin-1 at

$8431 \mu \mathrm{g} / \mathrm{mL}$ or ConA at $25 \mu \mathrm{g} / \mathrm{mL}$. After a 30 min incubation on ice, cells were washed twice in

844 FACS wash, then incubated with secondary F(ab')2 (for Fc-Dectin-1 only) on ice for 45

845 minutes, with a further 2 wash steps. Flow cytometry was performed on an LSR Fortessa 
846 cytometer (BD, Macquarie Park, Australia) with thanks to the University of Aberdeen IFCC

847 Core Facility. Data were analysed using FlowJo software (version 10.0.8). For the detailed

848 gating strategy see Extended Data Fig. 7b.

849

850 In vivo experimental model of disseminated candidiasis

851 For the in vivo experiments C. auris strain 10051895 was selected as representative of Clade I,

852 which was the first $C$. auris clade identified and associated with bloodstream infections and

853 high mortality rates ${ }^{17}$. Experiments were conducted with a total of 200 C57BI6 male mice, 7-8

854 weeks old, which were purchased from Pasteur Institute (Athens, Greece, EL 25 BIObr 011).

855 Mice were allowed to acclimate for seven days before start of the experiments. Animals were

856 housed in cages, with no more than 5 mice per cage, under constant temperature $\left(21^{\circ} \mathrm{C}\right)$ and

857 humidity with a 12-h light/dark cycle. All animals had ad libitum access to food and water.

858 Analgesia was achieved with paracetamol suppositories. Other analgesics were avoided in

859 order to avoid interactions with the immune system. Healthy mice were i.v. challenged via the

860 tail vein with $1 \times 10^{7} \mathrm{CFU} /$ mouse log-phase inoculum ${ }^{46,47}$ of $C$. albicans $10061110(\mathrm{n}=11)$ and C.

861 auris 10051895 ( $n=10)$ following slight anesthesia with methoxyflurane (2, 2-dichloro-1,1

862 difluoroethyl methyl-ether in butylated hydroxytoluene $0.01 \% \mathrm{w} / \mathrm{w})$. Mice were split into

863 groups via a randomization table. Survival was recorded for 14 days; three- and seven-days

864 post challenge mice were sacrificed by the intramuscular injection of ketamine. For evaluating

865 the fungal burden at day 3 and day 7 from the inoculation of $1 \times 10^{6} \mathrm{CFU} /$ mice $(\mathrm{n}=4-5$ mice per

866 group per timepoint), we removed kidneys and livers were weighted and homogenized. The

867 number of fungal counts were measured via serial dilutions 1:10 at 0.9\% saline and expressed

868 as $\log 10 \mathrm{CFU} / \mathrm{g}$. For collecting the organs homogenates, at day 3 and day 7 from the injection 
869 of $1 \times 10^{6}$ Candida CFU/mice ( $n=5-6$ mice per group per timepoint), after a midline incision

870 under aseptic conditions, the entire spleen was removed and segments of the right kidney

871 and of the liver were cut and put into separate sterile containers.

872

873 Statistical analysis

874 Statistical analysis, except when otherwise indicated, was performed using the Graphpad

875 Prism 7 software. All experiments were performed at least in duplicate. In experiments with a

876 sample size $<4$, no statistical testing was performed due to a small sample size. Datasets with

877 a sample size $>8$ were tested for normality via the D'Agostino-Pearson omnibus normality

878 test, and when normally distributed subjected to a 1-way ANOVA including Holm-Sidak's

879 multiple comparison test, as specified in corresponding method section and figure captions.

880 Given the experimental set-up, where the same donors were used, yet exposed to different

881 Candida species and strains (stimulations), measurements were considered

882 paired/dependent. Hence, the Wilcoxon signed-rank test for non-parametric matched data

883 was applied to non-normally distributed data. To enhance insight in the $C$. auris' induced host

884 response, all statistical analysis was performed comparing respective $C$. auris strains with $C$.

885 albicans, in the current study considered the control species. In the case of non-normally

886 distributed data and comparison to multiple control strains, a Kruskall Wallis test including

887 Dunn's multiple comparison was applied. For statistical testing of $I L-6, I L-1 B$, and IL-1RN

888 expression levels, an ordinary 1-way ANOVA was performed comparing the Log2 Fold

889 Changes and corresponding IfcSEs between the respective $C$. auris and $C$. albicans (live, $\beta$ -

890 glucans and mannans) conditions. Moreover, in vivo mice data presents an exception, with a 
891 Log rank-test for survival assessment and Mann Whitney $U$ test to assess fungal burden due 892 sample independence. In all cases, a p-value of $<0.05$ was considered significant. 
8941 Meis, J. F. \& Chowdhary, A. Candida auris: a global fungal public health threat. Lancet 895 Infect Dis, doi:10.1016/S1473-3099(18)30609-1 (2018).

8962 Clancy, C. J. \& Nguyen, M. H. Emergence of Candida auris: An International Call to 897 Arms. Clin Infect Dis 64, 141-143, doi:10.1093/cid/ciw696 (2017).

8983 de Groot, T., Puts, Y., Berrio, I., Chowdhary, A. \& Meis, J. F. Development of Candida 899 auris Short Tandem Repeat Typing and Its Application to a Global Collection of $900 \quad$ Isolates. mBio 11, doi:10.1128/mBio.02971-19 (2020).

9014 Lockhart, S. R. et al. Simultaneous Emergence of Multidrug-Resistant Candida auris on 9023 Continents Confirmed by Whole-Genome Sequencing and Epidemiological Analyses. 903 Clin Infect Dis 64, 134-140, doi:10.1093/cid/ciw691 (2017).

9045 Chow, N. A. et al. Potential Fifth Clade of Candida auris, Iran, 2018. Emerg Infect Dis $905 \quad 25,1780-1781$, doi:10.3201/eid2509.190686 (2019).

9066 Welsh, R. M., Sexton, D. J., Forsberg, K., Vallabhaneni, S. \& Litvintseva, A. Insights into 907 the Unique Nature of the East Asian Clade of the Emerging Pathogenic Yeast Candida 908 auris. J Clin Microbio/ 57, doi:10.1128/JCM.00007-19 (2019).

9097 Szekely, A., Borman, A. M. \& Johnson, E. M. Candida auris Isolates of the Southern $910 \quad$ Asian and South African Lineages Exhibit Different Phenotypic and Antifungal 911 Susceptibility Profiles In Vitro. J Clin Microbio/ 57, doi:10.1128/JCM.02055-18 (2019).

9128 Borman, A. M., Szekely, A. \& Johnson, E. M. Comparative Pathogenicity of United 913 Kingdom Isolates of the Emerging Pathogen Candida auris and Other Key Pathogenic 914 Candida Species. mSphere 1, doi:10.1128/mSphere.00189-16 (2016).

9159 Kathuria, S. et al. Multidrug-Resistant Candida auris Misidentified as Candida 916 haemulonii: Characterization by Matrix-Assisted Laser Desorption Ionization-Time of 
Flight Mass Spectrometry and DNA Sequencing and Its Antifungal Susceptibility Profile

918 Variability by Vitek 2, CLSI Broth Microdilution, and Etest Method. J Clin Microbio/ 53,

919 1823-1830, doi:10.1128/JCM.00367-15 (2015).

92010 Mizusawa, M. et al. Can Multidrug-Resistant Candida auris Be Reliably Identified in 921 Clinical Microbiology Laboratories? J Clin Microbiol 55, 638-640, 922 doi:10.1128/JCM.02202-16 (2017).

92311 Ruiz-Gaitan, A. et al. An outbreak due to Candida auris with prolonged colonisation 924 and candidaemia in a tertiary care European hospital. Mycoses 61, 498-505, 925 doi:10.1111/myc.12781 (2018).

92612 Schelenz, S. et al. First hospital outbreak of the globally emerging Candida auris in a $927 \quad$ European hospital. Antimicrob Resist Infect Control 5, 35, doi:10.1186/s13756-016$928 \quad 0132-5(2016)$

92913 Vallabhaneni, S. et al. Investigation of the First Seven Reported Cases of Candida auris, 930 a Globally Emerging Invasive, Multidrug-Resistant Fungus-United States, May 2013931 August 2016. Am J Transplant 17, 296-299, doi:10.1111/ajt.14121 (2017).

93214 Lee, W. G. et al. First three reported cases of nosocomial fungemia caused by Candida 933 auris. J Clin Microbiol 49, 3139-3142, doi:10.1128/JCM.00319-11 (2011).

93415 Welsh, R. M. et al. Survival, Persistence, and Isolation of the Emerging Multidrug935 Resistant Pathogenic Yeast Candida auris on a Plastic Health Care Surface. J Clin 936 Microbiol 55, 2996-3005, doi:10.1128/JCM.00921-17 (2017).

93716 Rudramurthy, S. M. et al. Candida auris candidaemia in Indian ICUs: analysis of risk 938 factors. J Antimicrob Chemoth 72, 1794-1801, doi:10.1093/jac/dkx034 (2017). 
93917 Chowdhary, A., Sharma, C. \& Meis, J. F. Candida auris: A rapidly emerging cause of 940 hospital-acquired multidrug-resistant fungal infections globally. PLoS Pathog 13, $941 \quad$ e1006290, doi:10.1371/journal.ppat.1006290 (2017).

94218 Al Maani, A. et al. Ongoing Challenges with Healthcare-Associated Candida auris 943 Outbreaks in Oman. J Fungi (Basel) 5, doi:10.3390/jof5040101 (2019).

94419 Arendrup, M. C., Chowdhary, A., Astvad, K. M. T. \& Jorgensen, K. M. APX001A In Vitro 945 Activity against Contemporary Blood Isolates and Candida auris Determined by the

946 EUCAST Reference Method. Antimicrob Agents Chemother 62, 947 doi:10.1128/AAC.01225-18 (2018).

94820 Hager, C. L. et al. In Vitro and In Vivo Evaluation of the Antifungal Activity of 949 APX001A/APX001 against Candida auris. Antimicrob Agents Chemother 62, 950 doi:10.1128/AAC.02319-17 (2018).

95121 Arendrup, M. C., Jorgensen, K. M., Hare, R. K. \& Chowdhary, A. EUCAST in vitro activity 952 of Ibrexafungerp (SCY-078) against C. auris isolates; comparison with activity against C.

953 albicans and C. glabrata and with that of six comparators. Antimicrob Agents $954 \quad$ Chemother, doi:10.1128/AAC.02136-19 (2019).

95522 Berkow, E. L., Angulo, D. \& Lockhart, S. R. In Vitro Activity of a Novel Glucan Synthase 956 Inhibitor, SCY-078, against Clinical Isolates of Candida auris. Antimicrob Agents 957 Chemother 61, doi:10.1128/AAC.00435-17 (2017).

95823 Larkin, E. et al. The Emerging Pathogen Candida auris: Growth Phenotype, Virulence 959 Factors, Activity of Antifungals, and Effect of SCY-078, a Novel Glucan Synthesis 960 Inhibitor, on Growth Morphology and Biofilm Formation. Antimicrob Agents 961 Chemother 61, doi:10.1128/AAC.02396-16 (2017). 
96224 Hager, C. L., Larkin, E. L., Long, L. A. \& Ghannoum, M. A. Evaluation of the efficacy of 963 rezafungin, a novel echinocandin, in the treatment of disseminated Candida auris 964 infection using an immunocompromised mouse model. J Antimicrob Chemother 73, 965 2085-2088, doi:10.1093/jac/dky153 (2018).

96625 Lepak, A. J., Zhao, M. \& Andes, D. R. Pharmacodynamic Evaluation of Rezafungin 967 (CD101) against Candida auris in the Neutropenic Mouse Invasive Candidiasis Model. 968 Antimicrob Agents Chemother 62, doi:10.1128/AAC.01572-18 (2018).

96926 Richardson, J. P. \& Moyes, D. L. Adaptive immune responses to Candida albicans $970 \quad$ infection. Virulence 6, 327-337, doi:10.1080/21505594.2015.1004977 (2015).

97127 Erwig, L. P. \& Gow, N. A. Interactions of fungal pathogens with phagocytes. Nat Rev $972 \quad$ Microbiol 14, 163-176, doi:10.1038/nrmicro.2015.21 (2016).

97328 Gow, N. A., van de Veerdonk, F. L., Brown, A. J. \& Netea, M. G. Candida albicans 974 morphogenesis and host defence: discriminating invasion from colonization. Nat Rev $975 \quad$ Microbiol 10, 112-122, doi:10.1038/nrmicro2711 (2011).

97629 Pathirana, R. U. et al. Fluconazole-Resistant Candida auris Is Susceptible to Salivary $977 \quad$ Histatin 5 Killing and to Intrinsic Host Defenses. Antimicrob Agents Chemother 62, 978 doi:10.1128/AAC.01872-17 (2018).

97930 Johnson, C. J., Davis, J. M., Huttenlocher, A., Kernien, J. F. \& Nett, J. E. Emerging Fungal 980 Pathogen Candida auris Evades Neutrophil Attack. MBio 9, doi:10.1128/mBio.01403$981 \quad 18$ (2018).

98231 Navarro-Arias, M. J. et al. Differential recognition of Candida tropicalis, Candida 983 guilliermondii, Candida krusei, and Candida auris by human innate immune cells. 984 Infect Drug Resist 12, 783-794, doi:10.2147/IDR.S197531 (2019). 
98532 Munoz, J. F. et al. Genomic insights into multidrug-resistance, mating and virulence in 986 Candida auris and related emerging species. Nat Commun 9, 5346, 987 doi:10.1038/s41467-018-07779-6 (2018).

98833 Brown, G. D. et al. Hidden killers: human fungal infections. Sci Transl Med 4, 165rv113, 989 doi:10.1126/scitransImed.3004404 (2012).

99034 Hall, R. A. \& Gow, N. A. Mannosylation in Candida albicans: role in cell wall function 991 and immune recognition. Mol Microbiol 90, 1147-1161, doi:10.1111/mmi.12426 992 (2013).

99335 Gow, N. A. et al. Immune recognition of Candida albicans beta-glucan by dectin-1. J 994 Infect Dis 196, 1565-1571, doi:10.1086/523110 (2007).

99536 Netea, M. G. et al. Immune sensing of Candida albicans requires cooperative 996 recognition of mannans and glucans by lectin and Toll-like receptors. J Clin Invest 116, 997 1642-1650, doi:10.1172/JCI27114 (2006).

99837 Klebanoff, S. J. Myeloperoxidase: friend and foe. J Leukoc Biol 77, 598-625, 999 doi:10.1189/jlb.1204697 (2005).

100038 Kerrigan, A. M. \& Brown, G. D. Syk-coupled C-type lectin receptors that mediate 1001 cellular activation via single tyrosine based activation motifs. Immunol Rev 234, 335$1002 \quad 352$, doi:10.1111/j.0105-2896.2009.00882.x (2010).

100339 Gringhuis, S. I. et al. Dectin-1 directs T helper cell differentiation by controlling 1004 noncanonical NF-kappaB activation through Raf-1 and Syk. Nat Immunol 10, 203-213, 1005 doi:10.1038/ni.1692 (2009).

100640 Yan, L. et al. Unique Cell Surface Mannan of Yeast Pathogen Candida auris with 1007 Selective Binding to IgG. ACS Infect Dis, doi:10.1021/acsinfecdis.9b00450 (2020). 
100841 Hall, R. A. \& Gow, N. A. R. Mannosylation in Candida albicans: role in cell wall function

1009 and immune recognition. Molecular Microbiology 90, 1147-1161 (2013).

101042 Marakalala, M. J. et al. Differential adaptation of Candida albicans in vivo modulates

1011 immune recognition by dectin-1. PLoS Pathog 9, e1003315,

1012 doi:10.1371/journal.ppat.1003315 (2013).

101343 Keppler-Ross, S., Douglas, L., Konopka, J. B. \& Dean, N. Recognition of Yeast by Murine

1014 Macrophages Requires Mannan but Not Glucan. Eukaryot Cell 9, 1776-1787 (2010).

101544 McKenzie, C. G. J. et al. Contribution of Candida albicans Cell Wall Components to 1016 Recognition by and Escape from Murine Macrophages. Infect Immun 78, 1650-1658 1017 (2010).

101845 Tucey, T. M. et al. Glucose Homeostasis Is Important for Immune Cell Viability during 1019 Candida Challenge and Host Survival of Systemic Fungal Infection. Cell Metab 27, 988$1020 \quad 1006$ e1007, doi:10.1016/j.cmet.2018.03.019 (2018).

102146 Fakhim, H. et al. Comparative virulence of Candida auris with Candida haemulonii, 1022 Candida glabrata and Candida albicans in a murine model. Mycoses 61, 377-382, 1023 doi:10.1111/myc.12754 (2018).

102447 Ben-Ami, R. et al. Multidrug-Resistant Candida haemulonii and C. auris, Tel Aviv, Israel. 1025 Emerg Infect Dis 23, doi:10.3201/eid2302.161486 (2017).

$102648 \quad$ Urban, C. F. \& Nett, J. E. Neutrophil extracellular traps in fungal infection. Semin Cell 1027 Dev Biol 89, 47-57, doi:10.1016/j.semcdb.2018.03.020 (2019).

102849 Urban, C. F., Reichard, U., Brinkmann, V. \& Zychlinsky, A. Neutrophil extracellular traps 1029 capture and kill Candida albicans yeast and hyphal forms. Cell Microbiol 8, 668-676, $1030 \quad$ doi:10.1111/j.1462-5822.2005.00659.x (2006). 
103150 Wiederhold, N. P. et al. Efficacy of Delayed Therapy with Fosmanogepix (APX001) in a 1032 Murine Model of Candida auris Invasive Candidiasis. Antimicrob Agents Chemother 63, 1033 doi:10.1128/AAC.01120-19 (2019).

103451 Oosting, M. et al. Borrelia-induced cytokine production is mediated by spleen tyrosine 1035 kinase (Syk) but is Dectin-1 and Dectin-2 independent. Cytokine 76, 465-472 (2015).

103652 Gaus, H., Miller, C. M., Seth, P. P. \& Harris, E. N. Structural Determinants for the 1037 Interactions of Chemically Modified Nucleic Acids with the Stabilin-2 Clearance 1038 Receptor. Biochemistry 57, 2061-2064, doi:10.1021/acs.biochem.8b00126 (2018).

103953 Sherrington, S. L. et al. Adaptation of Candida albicans to environmental pH induces 1040 cell wall remodelling and enhances innate immune recognition. PLoS Pathog 13, 1041 e1006403, doi:10.1371/journal.ppat.1006403 (2017).

104254 Kruppa, M., Greene, R. R., Noss, I., Lowman, D. W. \& Williams, D. L. C. albicans 1043 increases cell wall mannoprotein, but not mannan, in response to blood, serum and 1044 cultivation at physiological temperature. Glycobiology 21, 1173-1180, 1045 doi:10.1093/glycob/cwr051 (2011).

104655 Martin, M. Cutadapt Removes Adapter Sequences From High-Throughput Sequencing 1047 Reads. EMBnet.journa/ v. 17, doi:https://doi.org/10.14806/ej.17.1.200. (2016).

104856 Dobin, A. et al. STAR: ultrafast universal RNA-seq aligner. Bioinformatics 29, 15-21, 1049 doi:10.1093/bioinformatics/bts635 (2013).

105057 Anders, S., Pyl, P. T. \& Huber, W. HTSeq-a Python framework to work with high1051 throughput sequencing data. Bioinformatics 31, 166-169, 1052 doi:10.1093/bioinformatics/btu638 (2015). 
105358 Zhu, A., Ibrahim, J. G. \& Love, M. I. Heavy-tailed prior distributions for sequence count

1054 data: removing the noise and preserving large differences. Bioinformatics, 1055 doi:10.1093/bioinformatics/bty895 (2018).

105659 Kamburov, A., Wierling, C., Lehrach, H. \& Herwig, R. ConsensusPathDB--a database for 1057 integrating human functional interaction networks. Nucleic acids research 37, D623$1058 \quad$ 628, doi:10.1093/nar/gkn698 (2009).

105960 Kanehisa, M. \& Goto, S. KEGG: kyoto encyclopedia of genes and genomes. Nucleic 1060 acids research 28, 27-30, doi:10.1093/nar/28.1.27 (2000).

106161 Fabregat, A. et al. The Reactome Pathway Knowledgebase. Nucleic acids research 46, 1062 D649-d655, doi:10.1093/nar/gkx1132 (2018).

106362 Lowman, D. W. et al. Mannan structural complexity is decreased when Candida 1064 albicans is cultivated in blood or serum at physiological temperature. Carbohydr Res $1065 \quad 346,2752-2759$, doi:10.1016/j.carres.2011.09.029 (2011).

106663 Graus, M. S. et al. Mannan Molecular Substructures Control Nanoscale Glucan 1067 Exposure in Candida. Cell Rep 24, 2432-2442 e2435, doi:10.1016/j.celrep.2018.07.088 1068 (2018).

106964 Smith, A. J. et al. Immunoregulatory Activity of the Natural Product Laminarin Varies 1070 Widely as a Result of Its Physical Properties. J Immunol 200, 788-799, 1071 doi:10.4049/jimmunol.1701258 (2018).

1072

1073 DATA AVAILABILITY

1074 Requests for materials should be addressed to the corresponding autor (M.G.N.). The 1075 datasets generated from this study are accessible through GEO Series accession number 
is provided for main and extended data figures.

1078

1079

COMPETING INTERESTS

1080

The authors declare no competing interests.

1081

1082

ACKNOWLEDGMENTS

1083

We would like to thank Trees Jansen for performing the initial pilot experiments and Ilse

1084

Curfs-Breuker and Dirk Faro for their support at the CWZ hospital. We thank Charlotte Kaffa,

1085

BSc. for her technical assistance. We would like to thank Vinod Kumar for his input during the

transcriptomic data analysis, Mark Gresnigt for the in vitro experimental suggestions and

1087

Anouk Becker for the help during the revision experiments. AJPB and NARG thank the MRC

1088

(MR/M026663/1) and Wellcome for support and the Medical Research Council (MRC) Centre

1089

for Medical Mycology at the University of Aberdeen (MR/N006364/1). A.H. and S.K. were

1090

supported by the Radboud Institute for Molecular Life Sciences. Part of the study was

1091

supported by the Hellenic Institute for the Study of Sepsis. D.L.W. was supported by National

1092

Institutes of Health grants NIH GM083016, GM119197 and C06RR0306551. M.G.N. was

1093

supported by an ERC Advanced Grant (no. 833247) and a Spinoza Grant from the Netherlands

1094

Organization for Scientific Research.

1095

1096

AUTHOR CONTRIBUTIONS

1097 Conceptualization, J.F.M., D.L.W., M.G.N.; Methodology, M.B., S.K., J.M.B., M.J., D.R., M.D.K.,

1098 D.W.L., M.Z., Y.N.J., A.C., A.H., N.A.R.G., A.J.P.B., J.F.M., D.L.W., M.G.N.; Investigation, M.B.,

1099 S.K., J.M.B., M.J, D.R., D.W.L.,P.R., B.G., F.L.V., B.J.K., E.J.B., G.R., A.H, N.A.R.G, A.J.P.B., D.L.W. 
1102 D.L.W., M.G.N.; Supervision: M.J., F.L.V., A.H., N.A.R.G, A.J.P.B. and M.G.N. M.B. and S.K.

1103 contributed equally to this work. J.F.M., D.W.L. and M.G.N. share senior authorship.

1104

1105

1106

1107

1108

1109

1110

1111

1112

1113

1114

1115

1116

1117

1118

1119

1120

1121

1122

1123

1124

1125

1126

1127

1128

1129

1130

1131

1132

1133

1134

1135

\section{Main Figure Legends}

Figure 1 | Comparative analysis of the general and clade-specific $C$. auris induced host response with $C$. albicans at 24 hours. a, Venn diagram representing the number of DEG of both Candida species and their relative overlap, reveals substantial overlap between the C. albicans (10061110) and C. auris (KCTC17810, clade II) live induced host-response at 24 hours. DEG were subjected to a pathway enrichment analysis, in turn revealing the top 15 Candida intrinsic (overlapping DEG, middle panel) and species specific (DEG unique for $C$. albicans, left panel; DEG unique for $C$. auris, right panel) pathways. Enrichment determined using Consensus PathDB, including pathways as defined by KEGG (red) and Reactome (pink), considering a p-adjusted value $<0.01$ (indicated as 'q-value') significant. Size of the geometric points indicates the amount of DEG in relation to the pathways' size. The exact $q$ values and DEG in pathways can be found in Supplementary Table 2. b-c, C. auris is a more potent inducer of the immune system in comparison to $C$. albicans. b, TNF- $\alpha, I L-6, I L-1 \beta$, and IL-1Ra levels in supernatants of PBMCs after stimulation without (RPMI; negative control) or with live $C$. albicans and C. auris for 24 hours $(n=12)$. c, TNF- $\alpha, I L-6, I L-1 \beta$, and IL-1Ra levels in supernatants of PBMCs after stimulation without (RPMI; negative control) or with live $C$. albicans and $C$. auris from all five geographical clades for 24 hours $(n=8)$. Graphs represent mean \pm SEM, pooled from at least two independent experiments. ${ }^{*} p<0.05,{ }^{* *} p<0.01,{ }^{* * *} p<0.001$, two-sided Wilcoxon matched pairs signed-rank test was performed comparing respective $C$. auris strains with $C$. albicans as control or reference species. The data used to make this figure can be found in Source Data Fig.1.

Figure 2 | Evaluation of $C$. auris phagocytosis dynamics by human and murine host immune cells and heat-sensitivity of the cell wall component responsible for $C$. auris induced cytokine production. a, The BMDM phagocytic capacity of live $C$. albicans or $C$. auris strains in a 3-hour period. BMDM engulfment, depicted as the percentage of macrophages having phagocytosed at least one fungal cell (left). Phagocytic index was considered the number of fungal cells engulfed per 100 macrophages (right); graphs represent mean $(n=9)$, pooled from at least two independent experiments. b, Phagocytosis assay in human PBMCs: percentage of FITC-positive cells in the $\mathrm{CD}_{14}{ }^{+}$population. Phagocytosis efficiency assessed as percentage of $\mathrm{CD}_{1} 4^{+}$cells that engulfed FITC-labelled Candida (left) and corresponding mean fluorescence intensity (MFI) of the total $\mathrm{CD}_{14}{ }^{+}$population (right). Graphs represent mean $\pm \operatorname{SEM}(n=6)$, pooled from two independent experiments, $* p<0.05$, two-sided 
1136 Wilcoxon matched pairs signed-rank test, comparing respective $C$. auris strains with $C$. albicans as 1137 control or reference species. c, Distribution of phagocytosed live fungal cells per macrophage in a 1138 period of 3 hours, $n \geq 100$ observations per condition. d, Killing capacity of live C. albicans and C. auris, 1139 depicted as the percentage of lysed macrophages (BMDM) after 3 hours of exposure. 1140 Yeast:Macrophage ratio (MOI) was 3:1. Graphs represent mean \pm SEM, $n=9$ ( $n=10$ for $C$. auris 1141 10051895), pooled from at least two independent experiments, $* p<0.05$, Kruskall Wallis test with 1142 two-sided Dunn's multiple comparison between the two C. auris strains and the two C. albicans. e, 1143 TNF- $\alpha(n=10)$, IL-6 $(n=13)$, IL-1 $(n=13)$, and IL-1Ra $(n=9)$ levels in supernatants of PBMCs after 1144 stimulation without (RPMI; negative control) or with heat-killed C. albicans or C. auris for 24 hours. 1145 Graphs represent mean \pm SEM, data are pooled from at least two independent experiments. ${ }^{*} \mathrm{p}<$ $11460.05,{ }^{* *} p<0.01,{ }^{* * *} p<0.001$, two-sided Wilcoxon matched pairs signed-rank test, comparing 1147 respective $C$. auris strains with $C$. albicans as control or reference species. Data used to make this 1148 figure can be found in Source Data Fig. 2.

1150 Figure 3 | Mannans are fundamental for orchestrating the $C$. auris induced late host response. 1151 a, Split Venn diagrams indicating the number of DEG upon C. albicans 10061110 (left) and C. auris 1152 KCTC17810 (clade II; right) live stimulation on the left, with its respective overlap between exposure to 1153 the purified cell wall components $\beta$-glucan and mannan. Left split Venn diagram visualizes the early, 41154 hour response, and the right split Venn diagram reflects the late, 24-hour response. b, PBMC 1155 production of cytokines TNF- $\alpha$, IL-6, IL-1 $\beta$, and IL-1Ra after 24 hours stimulation without (RPMI; 1156 negative control) or with purified $\beta$-glucans from $C$. albicans and $C$. auris strains in the presence of $115710 \%$ human serum, $n=6$ ( $n=3$ for $C$. auris $\beta$-glucan 10051244). c, PBMC production of TNF- $\alpha, I L-6, I L-$ $11581 \beta$, and IL-1Ra after 24-hour stimulation without (RPMI; negative control) or with Pam3cys and/or 1159 purified $\beta$-glucans from different $C$. albicans and $C$. auris strains in the presence of $10 \%$ human serum, $1160 \mathrm{n}=6 . \mathrm{d}, \mathrm{PBMC}$ production of cytokines TNF- $\alpha, \mathrm{IL}-6, \mathrm{IL}-1 \beta$, and IL-1Ra after 24 hours stimulation without 1161 (RPMI; negative control) or with purified mannans from C. albicans and C. auris strains in the presence 1162 of $10 \%$ human serum, $n=10$. Graphs represent mean \pm SEM, pooled from at least two independent 1163 experiments. ${ }^{*} p<0.05,{ }^{* *} p<0.01,{ }^{* * *} p<0.001$, two-tailed Wilcoxon matched pairs signed-rank 1164 test and was performed comparing both cell wall components extracted from $C$. auris strains with $C$. 1165 albicans as control or reference species (SC5314). Data used to make this figure can be found in 1166 Source Data Fig. 3.

1167 Figure 4 | Structural characterization of $C$. auris mannans. (A) 2D COSY Nuclear magnetic resonance 1168 (NMR) spectroscopy analysis of mannans purified from various C. auris clinical strains, originating from 1169 clades I, II and IV, and C. albicans. Although NMR reveals varying side-chain lengths containing $\alpha-1,2-$ 1170 mannose, $\alpha-1,3$-mannose and $\beta-1,2$-mannose across clinical isolates, characteristic for $C$. auris 1171 mannans are the two distinct $M-\alpha-1$-phosphate side chains.

1172 Figure 5 | Exploration of PRR and signaling pathways involved in the $C$. auris induced host cytokine 1173 production.

1174 a, PBMC production of cytokines TNF- $\alpha$, IL-6, IL-1 $\beta$, and IL-1Ra after 24 hours stimulation without 1175 (RPMI; negative control) or with PFA-fixed C. albicans and C. auris strains, subjected to vehicle (DMSO) $1176(n=9)$ or a 1-hour pre-incubation with Syk $(n=6)$ and Raf-1 $(n=9)$ inhibitors. b, PBMC production of 
cytokines TNF- $\alpha$, IL-6, IL-1 $\beta$, and IL-1Ra after 24 hours stimulation without (RPMI; negative control) or

1178 with live $C$. albicans and $C$. auris strains, subjected to a 1 hour pre-incubation with the isotype

1179 antibodies IgG2b, Goat IgG and IgG1, or DC-SIGN, Dectin-1, Mincle, MMR, CR3 and Dectin-2 blocking

1180 antibodies, $n=6$. Graphs represent mean \pm SEM, data pooled from at least two independent

1181 experiments, $* p<0.05, * * p<0.01$, a two-sided Wilcoxon matched pairs signed-rank test comparing

1182 (within each Candida strain) the respective inhibitor with its vehicle, $\mathrm{b}$ two-sided Wilcoxon matched

1183 pairs signed-rank test comparing (within each Candida strain) the neutralizing antibodies with the

1184 correspondent isotype controls. Data used to make this figure can be found in Source Data Fig. 5.

1185 Figure 6 | C. auris is less virulent than C. albicans in an experimental model of murine disseminated

1186 candidiasis. a, Survival curve of immunocompetent mice i.v. challenged with $C$. albicans $(n=11)$ or $C$.

1187 auris $(\mathrm{n}=10)$. Mice were i.v. injected with $1 \times 10^{7} \mathrm{CFU}$ of the respective Candida strain and monitored

1188 daily. b, Fungal burden of immunocompetent mice i.v. challenged with $1 \times 10^{6} \mathrm{CFU}$ of $C$. albicans (day 3 ,

$1189 \mathrm{n}=5$; day $7, n=4$ ) or $C$. auris (day 3, n=5; day 7, n=5) in the liver and kidney at 3 (top) and 7 (bottom)

1190 days post injection. $\mathrm{c}-\mathrm{d}, \mathrm{KC}$ (c) and MPO (d) production in supernatants from liver, kidney and spleen

1191 homogenates ( $n=6$ per group per time-point). e, KC production in plasma of mice ( $n=6$ per group per

1192 time-point) infected i.v. with $1 \times 10^{6}$ CFU of $C$. albicans or C. auris. Graphs represent mean \pm SEM, data

1193 pooled from at least two independent experiments, ${ }^{*} p<0.05,{ }^{* *} p<0.01,{ }^{* * *} p<0.001$, a, Mantel-

1194 Cox two-sided log-rank test, b-e two-sided Mann-Whitney $U$ test.

$1195 \mathrm{f}$, Ratio of the mean MPO or KC production (log scale, data from Figure $6 d, 6 c$ ) to the mean of fungal

1196 burden (log scale, data from Figure 6b) in kidney (blue bars) and liver (white bars) of mice infected

1197 with C. albicans or C. auris. Data are represented as the ratio of the mean log MPO and KC values ( $n=6$

1198 per group per time-point), to the mean $\log \mathrm{CFU}$ (C. albicans: day 3, $n=5$; day $7, n=4$. C. auris: day 3,

$1199 n=5 ;$ day 7, n=5). Data used to make this figure can be found in Source Data Fig. 6.

1200 